\title{
Systematic search for extremely metal poor galaxies in the Sloan Digital Sky Survey
}

\author{
A. B. Morales-Luis ${ }^{1,2}$, J. Sánchez Almeidaa ${ }^{1,2}$, J. A. L. Aguerri1 ${ }^{1,2}$, and C. Muñoz-Tuñón ${ }^{1,2}$ \\ abml@iac.es, jos@iac.es, cmt@iac.es, jalfonso@iac.es
}

\begin{abstract}
We carry out a systematic search for extremely metal poor (XMP) galaxies in the spectroscopic sample of Sloan Digital Sky Survey (SDSS) data release 7 (DR7). The XMP candidates are found by classifying all the galaxies according to the form of their spectra in a region $80 \AA$ wide around $\mathrm{H} \alpha$. Due to the data size, the method requires an automatic classification algorithm. We use k-means. Our systematic search renders 32 galaxies having negligible [NII] lines, as expected in XMP galaxy spectra. Twenty one of them have been previously identified as XMP galaxies in the literature - the remaining eleven are new. This was established after a thorough bibliographic search that yielded only some 130 galaxies known to have an oxygen metallicity ten times smaller than the Sun (explicitly, with $12+\log (\mathrm{O} / \mathrm{H}) \leq 7.65)$. XMP galaxies are rare; they represent $0.01 \%$ of the galaxies with emission lines in SDSS/DR7. Although the final metallicity estimate of all candidates remains pending, strong-line empirical calibrations indicate a metallicity about one-tenth solar, with the oxygen metallicity of the twenty one known targets being $12+\log (\mathrm{O} / \mathrm{H}) \simeq 7.61 \pm 0.19$. Since the SDSS catalog is limited in apparent magnitude, we have been able to estimate the volume number density of XMP galaxies in the local universe, which turns out to be $(1.32 \pm 0.23) \cdot 10^{-4} \mathrm{Mpc}^{-3}$. The XMP galaxies constitute $0.1 \%$ of the galaxies in the local volume, or $\sim 0.2 \%$ considering only emission line galaxies. All but four of our candidates are blue compact dwarf galaxies (BCDs), and 24 of them have either cometary shape or are formed by chained knots.
\end{abstract}

Subject headings: methods: data analysis - galaxies: abundances - galaxies: formation - galaxies: starburst - galaxies: statistics

\footnotetext{
${ }^{1}$ Instituto de Astrofísica de Canarias, E-38205 La Laguna, Tenerife, Spain

${ }^{2}$ Departamento de Astrofísica, Universidad de La Laguna, Tenerife, Spain
} 


\section{Introduction}

The evolution of galaxies involves the birth and death of stars, therefore, galaxies unavoidably produce metals as they live. Thus, galaxies with very low metallic content are probably unevolved objects, and if we find them nearby, they provide a readily accessible fossil record from the early universe. These objects are to be expected according to the paradigm of hierarchical galaxy formation, where large galaxies arise through the assembly of smaller ones in an inefficient process leaving many dwarf galaxies as remnant (e.g., Klypin et al. 1999; Balogh et al. 2001; Diemand et al. 2007). They seem to be materialized as the extremely metal-poor (XMP) dwarf galaxies observed today which, consequently, would be the closest examples we can find of these elementary primordial units from which larger galaxies assembled (e.g., Izotov \& Thuan 2004a, 2007). Those units must have been extremely common in the past, but they cannot be directly observed at high redshift. Nearby low metallicity galaxies offer a chance for detailed studies otherwise impossible. Studies of their interstellar medium (ISM) can shed light on the properties of the primordial ISM at the time of galaxy formation (Izotov \& Thuan 2007). For example, even the most metal-deficient galaxies in the local universe formed from matter already enriched by an early star formation episode, and the determination of the minimum galactic metallicity seems to be the best constraint available on these first stars (e.g., Bromm \& Larson 2004; Thuan \& Izotov 2005). Because they have not undergone much chemical evolution, these galaxies are also the best objects for the determination of the primordial He abundance that constrains cosmological models (e.g., Peimbert \& Torres-Peimbert 1974; Pagel et al. 1992; Izotov \& Thuan 2004b).

The class of blue compact dwarf galaxies (BCDs; e.g., Cairós et al. 2001b, a; ; Amorín et al. 2007, 2009) contains the galaxies with the lowest known gas-phase metallicity (e.g., Kunth \& Östlin 2000; Papaderos et al. 2008). It is so far unclear whether such preference for XMP galaxies to be BCDs is genuine or if the association results from an observational bias that systematically disfavors low surface brightness objects. The best XMP candidates in the local universe are BCDs, but metal poor galaxies are found among other types of dwarf galaxies as well (see Kunth \& Östlin 2000).

Unfortunately, XMP galaxies are rare. The review by Kunth \& Östlin (2000) cites only 31 targets with metallicity below one tenth the solar value, which is the threshold customary used to define XMP galaxies. For decades I Zw 18 held the record of lowest metallicity (Sargent \& Searle 1970), and although a few other examples have been recently found (e.g., Izotov et al. 2005, 2009), there is a minimum metallicity close to that of I Zw 18, which corresponds to a few hundredths the solar value. The existence of such metallicity threshold is suggestive of the pre-galactic origin of metals as it happens with halo stars (e.g., Spite \& Spite 1982), but it may also be due to other effects like the early self contamina- 
tion of the ongoing starburst that rises any original level to a minimum metallicity (e.g., Kunth \& Sargent 1986), or even the technical difficulty of metallicity determinations below a threshold (Papaderos et al. 2008). The number of low metallicity galaxies has significantly increased since the work by Kunth \& Östlin (2000), but they are still rare objects. The thorough bibliographic compilation described in $\S 4$ shows only 129 such targets. The shortage of low metallicity galaxies is partly a consequence of their low luminosity as expected from the luminosity-metallicity relationship (e.g., Lequeux et al. 1979; Skillman et al. 1989; Tremonti et al. 2004). They must be faint and so detectable only within a very local volume.

In order to enlarge the list of this rare yet interesting objects, we have carried out an automatic search for low metallicity galaxies in the seventh Sloan Digital Sky Survey data release (SDSS/DR7). The work is reported here. So far as we are aware of, this is the first systematic search of this kind on SDSS/DR7, even though extensive searches in earlier SDSS data releases have been reported (e.g., Izotov et al. 2006a; Guseva et al. 2009). The advantage of an orderly search rather than the more traditional serendipitous discovery is twofold. First, it maximizes the number of potential candidates. Second, the bias of the selection is quantifiable, allowing us to estimate for the first time the volume number density of XMP galaxies in the local universe.

Low metallicity galaxies are characterized by having a [NII] $\lambda 6583$ line negligibly small as compared to $\mathrm{H} \alpha$. Thus, the ratio between $[\mathrm{NII}] \lambda 6583$ and $\mathrm{H} \alpha$ is used to measure metallicities through the appropriate calibration (e.g., Denicoló et al. 2002; Pettini \& Pagel 2004). A low value of this ratio has been imposed as a necessary condition in classical works seeking for XMP galaxies (e.g., Izotov et al. 2006a). Building on this classical approach, we address the problem in an original way by automatically classifying the galaxies according to the shape of their spectra in a region around $\mathrm{H} \alpha$. We expected the classification to automatically separate classes of galaxies whose spectra present this property, and those targets would be regarded as metal poor candidates. Note that our approach does not require the detailed knowledge of the spectral properties of the XMP galaxies, e.g., we do not have to specify a particular ratio [NII] $\lambda 6583$ over $\mathrm{H} \alpha$ for a galaxy to be included. They are determined by the classification algorithm in view of the existing spectra. This minimum need of prior knowledge makes the search novel and robust against uncertainties in the selection criteria. The above conjecture turned out to work, and the result of the study is presented here. We employ a robust classification algorithm called k-means, commonly used in data-mining (e.g., Everitt 1995), and which we have already successfully applied to sort out different types of astronomical spectra spanning from polarization profiles in the Sun (Sánchez Almeida \& Lites 2000; Viticchié \& Sánchez Almeida 2011) to galaxy spectra (Sánchez Almeida et al. 2009, 2010).

The paper is organized as follows. First, we describe the systematic search for low 
metallicity galaxies ( $\S 2$ ). SDSS/DR7 renders 32 XMP candidates. The physical properties of the galaxies thus selected are analyzed in $\S 3$. These candidates, together with the rest of SDSS/DR7 galaxies, allow us to compute the volume number density of low metallicity galaxies in the local universe $(\S$ 5). In order to contextualize our work, we carried out a comprehensive search for XMP in the literature. The results are given in $\S$ 4. A summary with conclusions and follow up work is provided in $\S 6$.

\section{K-means based search for galaxies of low metallicity}

As we mention in the introduction, the spectrum around $\mathrm{H} \alpha$ is very sensitive to metallicity. The ratio between the equivalent widths of [NII] $\lambda 6583$ and $\mathrm{H} \alpha$ goes to zero with decreasing metallicity, as calibrated by, e.g., Denicoló et al. (2002) or Pettini \& Pagel (2004). We make use of this sensitivity to select low metallicity galaxies classifying all the galaxies with spectra in SDSS/DR7 according to their shape around $\mathrm{H} \alpha$. Those classes containing spectra where $[\mathrm{NII}] \lambda 6583$ turns out to be negligible small with respect to $\mathrm{H} \alpha$ will be saved as candidates. For detailed information on the SDSS spectral catalog, see Stoughton et al. (2002a), Abazajian et al. (2009), as well as the comprehensive SDSS/DR7 web site1. The main properties of the catalog affecting our analysis are the spectral resolution of the spectra, some 2000 at $\mathrm{H} \alpha$, and the fact that it includes all galaxies up to an integrated $r$ magnitude of 17.8 .

Since the classification must be based on the shape of the spectrum rather that on other incidental property (e.g., the galaxy luminosity), the spectra must go through a previous normalization procedure. The original spectra are shifted to restframe wavelength using SDSS redshifts, and then normalized to the flux in the $g$ color filter. In addition, the continuum around $\mathrm{H} \alpha$ is subtracted, and this spectrum is re-normalized to the peak intensity of $[\mathrm{NII}] \lambda 6583$. Specifically, if $I(\lambda)$ stands for the spectrum in restframe wavelengths and normalized to $g$, then the spectrum to be classified is $S(\lambda)$,

$$
S(\lambda)=\frac{I(\lambda)-I_{c}(\lambda)}{\left|I(6583 \AA)-I_{c}(6583 \AA)\right|},
$$

where $\lambda$ stands for the wavelength, and $I(6583 \AA)$ is the intensity at the extreme of $[\mathrm{NII}] \lambda 6583$, i.e., the maximum if the line is in emission and the minimum if it is in absorption. The continuum intensity $I_{c}(\lambda)$ is derived by linear interpolation of the spectrum in two clean windows at both sides of the spectral region of interest, namely, from $6400 \AA$ to $6460 \AA$, and from

${ }^{1}$ http: //www . sdss .org/dr7 
$6610 \AA$ to $6670 \AA$. Note that the denominator of equation (1) is always positive so that the lines in absorption remain in absorption after normalization. We came across this normalization after some alternative trials. The normalization to [NII] $\lambda 6583$ turns out to maximize the contrast since it goes to zero for the low metallicity galaxies we are trying to identify. Figure 1 summarizes the normalization procedure. It includes the original spectrum (the black solid line) as well as the two continuum windows used to determine the continuum intensity. After normalization (i.e., after continuum removal plus division by the maximum intensity at $[\mathrm{NII}] \lambda 6583)$ it becomes the dashed line, which is the $80 \AA$ wide spectral range undergoing classification.

We employ the algorithm k-means for classification. It treats the spectra as vectors in a $n$-dimensional space, with $n$ the number of wavelengths. It is a rather standard technique in data-mining, machine learning, and artificial intelligence (e.g., Everitt 1995; Bishop 2006), and we have already successfully employed it for massive classification of galaxy spectra (Sánchez Almeida et al. 2008, 2010). It works iteratively, starting from randomly chosen cluster centers. Each galaxy spectrum is assigned to the cluster center that is closest in a least-squares sense. Then the cluster centers are recomputed as the average spectrum of all the spectra in the class, and the assignation begins again. The algorithm ends when no spectra are re-classified in two successive iterations. The main advantages are: (1) it is fast, simple, and robust, as requited to classify large data sets, (2) it guarantees that similar spectra end up in the same class, (3) it automatically yields the number of classes, and (4) it provides spectra that are characteristic of the classes. These template spectra are just the average of all the spectra in the class, and they can be analyzed and interpreted as individual galaxies under the same assumptions followed when applying the popular stacking technique (e.g., Ellison et al. 2000). The drawbacks are: (1) the starting random seeds influence the classification, and such effect must be followed up and, eventually, corrected for, and (2) the classes do not necessarily represent individual clusters in the classification space, but may be parts of clusters. The latter downside is not a problem in our particular application since we are not interested in clustering but in separating spectra with different shapes. As for the former, one have to carry out several independent trials to test the robustness of the classes on the initialization.

We apply k-means to the galaxies with spectra having redshift $\leq 0.25$, which is equivalent to classifying all the $\sim 9 \cdot 10^{5}$ galaxies with spectra in SDSS/DR7 since the low metallicity targets are expected to be faint ( $\S$ 1) and, consequently they cannot be observed at high redshift given the apparent magnitude threshold imposed by SDSS. (For a galaxy at redshift $\geq 0.25$ to be observed spectroscopically, the absolute $r$ magnitude must be smaller than -22.3.) The k-means procedure is applied in two successive steps, because a single application renders classes too coarse to separate low metallicity galaxies. In the first application, 
we remove classes having $\mathrm{H} \alpha$ in absorption, as well as those whose [NII] $\lambda 6583$ is much too large. Actually the limit is not imposed directly on $[\mathrm{NII}] \lambda 6583$, but on the equivalent oxygen abundance deduced via the so-called N2-method (e.g., Denicoló et al. 2002; Shi et al. 2005) which depends, exclusively, on the ratio between the equivalent widths of $[\mathrm{NII}] \lambda 6583$ and $\mathrm{H} \alpha$,

$$
\mathrm{N} 2=\log \left(W_{[\mathrm{NII}] \lambda 6583} / W_{\mathrm{H} \alpha}\right) .
$$

In the calibration by Pettini \& Pagel (2004), the oxygen abundance is,

$$
12+\log (\mathrm{O} / \mathrm{H}) \simeq 8.90+0.57 \mathrm{~N} 2
$$

or alternatively,

$$
12+\log (\mathrm{O} / \mathrm{H}) \simeq 9.37+2.03 \mathrm{~N} 2+1.26 \mathrm{~N} 2^{2}+0.32 \mathrm{~N} 2^{3}
$$

Galaxies belonging to classes whose template spectrum has $12+\log (\mathrm{O} / \mathrm{H}) \leq 8.2$ were used for a second k-means run. This arbitrary threshold was chosen as a trade off that removes enough high metallicity objects, yet allows the second classification to choose from a broad enough pool. The subset undergoing the second classification contains some 5000 galaxies, which correspond to only $0.6 \%$ of the original set. This second classification renders classes with a spectrum characteristic of low metallicity, i.e., with $\mathrm{H} \alpha \gg[\mathrm{NII}] \lambda 6583$. Several templates of the classes thus obtained are included in Fig. 2, It shows genuine low metallicity classes (e.g., class \# 14) as well as some others where the SDSS skyline removal pipeline has artificially cut out $[\mathrm{NII}] \lambda 6583$ (e.g., class \# 15). The finding of these fake low metallicity galaxies shows a weakness of the searching technique - some SDSS/DR7 galaxies with negligible [NII] 65583 may not be low metallicity after all, and the selection must be refined even further. However, this downside incidentally proves the procedure to be working properly, since we know it managed to identify spectra without $[\mathrm{NII}] \lambda 6583$. The problem arises as to how to separate genuine from fake low metallicity galaxies. Fortunately, it can be sorted out easily as we explain below. Figure 3 shows the oxygen abundance corresponding to the templates of the classes inferred from this second step, discarding those classes where the template shows obvious malfunctioning of the SDSS pipeline (i.e., like class \# 15 in Fig. 2). They are based on the two slightly different calibrations by Pettini \& Pagel (2004) given in equations (3) and (4). We use it to select the galaxies in classes \# 11 and \# 14 as XMP galaxy candidates. The selection criteria are somewhat arbitrary but we choose these two classes because (1) the metallicity estimated for the template using the N2-method are smaller than one-tenth solar 2 , which is the reference value for XMP galaxies ( $\S 1$ ), (2) there

\footnotetext{
${ }^{2}$ Or $W_{\mathrm{H} \alpha} / W_{\text {[NII] } \lambda 6583} \geq 140$, using the calibration in equation (4) and assuming $12+\log (\mathrm{O} / \mathrm{H})_{\odot}=8.65$ (Asplund 2005), as invoked by Pettini \& Pagel (2004).
} 


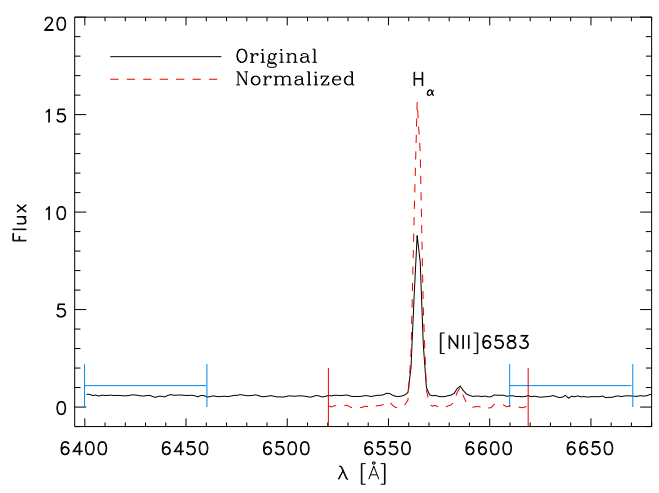

Fig. 1. - Spectral region classified to search for metal poor galaxies, which should have [NII] 66583 small compared to the nearby $\mathrm{H} \alpha$ line. The original spectrum (the black solid line) is normalized before classification rendering the (red) dashed line. The normalization includes removing the continuum, and dividing the residual by the maximum intensity of $[\mathrm{NII}] \lambda 6583$. The blue solid bars at the extremes mark the intervals used to determine the continuum. The central $80 \AA$ wide bar embraces the spectral region of interest. Wavelengths $\lambda$ are in $\AA$, and the spectra are given in arbitrary units.
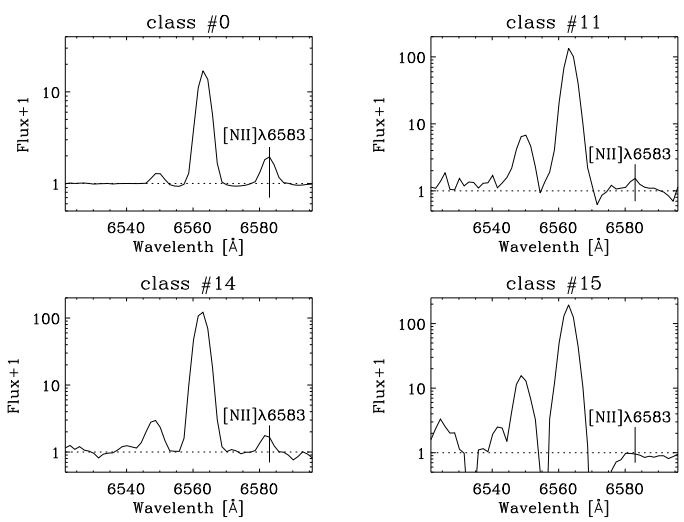

Fig. 2.- Several templates of the k-means classification characterized by having $\mathrm{H} \alpha \gg$ $[\mathrm{NII}] \lambda 6583$, which may correspond to classes of low metallicity galaxies. The individual spectra have their continua removed, and have been normalized to the peak intensity of $[\mathrm{NII}] \lambda 6583$. In order to be plotted in a logarithm scale, the spectra have been artificially uplift by one unit. These classes include spectra where the SDSS pipeline has artificially removed [NII] $\lambda 6583$ creating a mock low metallicity galaxy (e.g., class \# 15 is mostly formed by these spectra). Wavelengths are given in $\AA$. 
seems to be a gap in metallicity between these two classes and the rest (see Fig. 3), and (3) the number of galaxies the classes contain is small enough to allow a detailed inspection of the individual spectra. The original 49 spectra included in these two classes were visually inspected to discover that 20 of them were faults produced by the SDSS pipeline. These spectra are easily recognized because the pipeline linearly interpolates the spectrum around $[\mathrm{NII}] \lambda 6583$, and the presence of such a straight line boldly contrasts with the rest of the spectrum shape. They were excluded. Since faulty spectra ended up in low-metal classes, we checked the faulty classes for truly XMP spectra sneaked in. Most of the spectra were indeed failures from the pipeline, but we rescued three particularly low [NII] 26583 spectra included in these classes. All in all, our selection rendered 32 galaxies. Their coordinates and the unique identifier of the galaxy spectrum in SDSS/DR7 are listed in Table 1, The table also includes a column with the oxygen metallicity inferred applying equation (44) to the equivalent widths measured on the individual SDSS spectra. These values are only tentative since the prescription has an intrinsic scatter as large as 0.3 dex for individual galaxies (e.g., Pettini \& Pagel 2004; Shi et al. 2005; Pérez-Montero \& Contini 2009). Keeping this caveat in mind, the mean $12+\log (\mathrm{O} / \mathrm{H})$ of the candidates is 7.60 , with a standard deviation of 0.22 . As we will see in $\S$ 4, this estimate is in agreement with the metallicity of the subset of candidates whose abundance has been derived with more precise means.

K-means renders final classes that depend on the random initialization. This problem is not particularly severe in our application, since we are not trying to find clusters. We just try to separate spectra with a particular shape, independently on whether they appear on a single class or in several classes. Therefore, we did not expected the random initialization to represent a serious problem, yet we study the impact on the selected spectra by repeating the k-means classification 5 times. Classes with one-tenth solar metallicity are always present. They contain most of the 49 spectra in the original classification purged later on. Specifically, the two classes of lowest metallicity always include between $79 \%$ and $93 \%$ of the spectra in the classification.

Our search is systematic. If SDSS/DR7 has spectra with $\mathrm{H} \alpha \gg[\mathrm{NII}] \lambda 6583$, they will appear in one of the classes. The assumption that we detect all the spectra with this property is used in $\S 5$ to estimate the number density of XMP galaxies in the local universe. Deviations from this assumption are also analyzed in $\S$.

\section{Properties of the XMP candidates}

As pointed out in $\S$ 1, the known XMP galaxies tend to be BCD galaxies, which are

characterized by blue colors, high surface brightness, and low luminosity. Obviously, the 
Table 1. XMP candidates found by classifying all SDSS/DR7 galaxies according to their spectra around $\mathrm{H} \alpha$.

\begin{tabular}{|c|c|c|c|c|c|c|c|}
\hline \multirow[t]{2}{*}{ Index ${ }^{a}$} & \multirow[t]{2}{*}{ Name } & \multirow[t]{2}{*}{$g$} & \multirow[t]{2}{*}{ Redshift } & \multicolumn{2}{|c|}{$12+\log (\mathrm{O} / \mathrm{H})$} & \multirow[t]{2}{*}{ SpecObjID ${ }^{\mathrm{b}}$} & \multirow[t]{2}{*}{ Comment $^{\mathrm{c}}$} \\
\hline & & & & $\mathrm{d}$ & $\mathrm{e}$ & & \\
\hline 1 & SDSS J003630.40+005234.6 & 18.8 & 0.028 & 7.64 & - & 194442194734546944 & single knot \\
\hline $2^{\star}$ & SDSS J012534.19+075924.4 & 18.4 & 0.010 & 7.60 & 7.60 & 655785970125242368 & knotted cometary \\
\hline 3 & SDSS J015809.39+000637.2 & 18.1 & 0.012 & 7.75 & - & 302812946306695168 & cometary \\
\hline $4^{\star}$ & SDSS J030331.27-010947.1 & 19.7 & 0.030 & 7.48 & 7.22 & 225967511814799360 & cometary \\
\hline 5 & SDSS J031300.05+000612.1 & 19.2 & 0.029 & 7.82 & - & 299996706848636928 & cometary \\
\hline $6^{\star}$ & SDSS J080840.85+172856.4 & 19.2 & 0.044 & 7.36 & 7.48 & 585978593608204288 & single knot \\
\hline 7 & SDSS J082540.45+184617.2 & 19.0 & 0.038 & 7.75 & - & 640023302407979008 & single knot \\
\hline 8 & SDSS J084236.58+103313.9 & 17.7 & 0.011 & 7.58 & - & 724467307371298816 & cometary \\
\hline $9^{\star}$ & SDSS J093402.03+551427.7 & 16.4 & 0.002 & 6.88 & 7.17 & 156443095175528448 & 2-knot cometary \\
\hline 10 & SDSS J094254.27+340411.8 & 19.1 & 0.023 & 7.67 & - & 547698126572486656 & cometary? \\
\hline 11 & SDSS J100348.66+450457.7 & 17.5 & 0.009 & 7.65 & - & 265375788289753088 & single knot \\
\hline $12^{\star}$ & SDSS J101624.52+375445.9 & 15.9 & 0.004 & 7.61 & 7.58 & 401892408779866112 & cometary \\
\hline $13^{\star}$ & SDSS J103137.28+043422.0 & 16.2 & 0.004 & 7.52 & 7.70 & 162635977557278720 & cometary \\
\hline $14^{\star}$ & SDSS J104457.80+035313.1 & 17.5 & 0.013 & 7.01 & 7.44 & 162917331083722752 & cometary \\
\hline $15^{\star}$ & SDSS J111934.34+513012.1 & 16.8 & 0.004 & 7.75 & 7.51 & 247359886311030784 & 2-knot cometary \\
\hline 16 & SDSS J114506.25+501802.4 & 17.8 & 0.006 & 7.71 & - & 272412374642720768 & cometary \\
\hline $17^{\star}$ & SDSS J115132.94-022222.0 & 16.7 & 0.004 & 7.58 & 7.78 & 93111671593107456 & 2-knot cometary \\
\hline 18 & SDSS J115754.18+563816.7 & 16.9 & 0.001 & 7.83 & - & 369803376499621888 & cometary \\
\hline $19^{\star}$ & SDSS J120122.31+021108.3 & 17.6 & 0.003 & 7.69 & 7.49 & 145464500043120640 & cometary \\
\hline $20^{\star}$ & SDSS J121402.48+534517.4 & 17.3 & 0.003 & 7.54 & 7.64 & 287049377199947776 & cometary \\
\hline $21^{\star}$ & SDSS J123048.60+120242.8 & 16.7 & 0.004 & 7.78 & 7.73 & 454810434122285056 & cometary \\
\hline $22^{\star}$ & SDSS J125526.07-021334.0 & 19.1 & 0.052 & 7.73 & 7.83 & 95080395053203456 & single knot \\
\hline $23^{\star}$ & SDSS J132347.46-013252.0 & 18.1 & 0.023 & 7.21 & 7.78 & 96204976459612160 & single knot \\
\hline 24 & SDSS J132723.29+402204.1 & 19.0 & 0.012 & 7.67 & - & 412307391283986432 & single knot \\
\hline $25^{\star}$ & SDSS J133126.91+415148.3 & 17.1 & 0.012 & 7.64 & 7.75 & 412307391565004800 & cometary? \\
\hline $26^{\star}$ & SDSS J135525.66+465151.3 & 19.2 & 0.028 & 7.86 & 7.63 & 361921789577658368 & cometary? \\
\hline 27 & SDSS J141851.13+210239.7 & 17.6 & 0.009 & 7.64 & - & 784423532984532992 & cometary \\
\hline $28^{\star}$ & SDSS J142342.88+225728.7 & 17.8 & 0.033 & 7.67 & 7.72 & 600334402043510784 & single knot \\
\hline $29^{\star}$ & SDSS J150934.17+373146.1 & 17.3 & 0.033 & 7.74 & 7.85 & 394011865673367552 & cometary? \\
\hline $30^{\star}$ & SDSS J164710.66+210514.5 & 17.2 & 0.009 & 7.74 & 7.75 & 442143986740625408 & knotted cometary \\
\hline $31^{\star}$ & SDSS J223831.12+140029.7 & 18.9 & 0.021 & 7.64 & 7.45 & 533060792673632256 & 2-knot cometary \\
\hline $32^{\star}$ & SDSS J230210.00+004938.8 & 18.7 & 0.033 & 7.57 & 7.71 & 190784502518251520 & 2-knot cometary \\
\hline
\end{tabular}

aThose indexes marked with an asterisk correspond to known XMP galaxies. The rest are new.

${ }^{\mathrm{b}}$ Unique identifier of the galaxy spectrum in SDSS/DR7.

${ }^{\mathrm{c}}$ Sketch of galaxy shape.

${ }^{\mathrm{d}}$ Using the calibration by Pettini \& Pagel (2004), given in our equation (4).

${ }^{\mathrm{e}}$ From the literature as listed in Table 2 
metal-poor galaxies represent only a small fraction of the BCDs. In order to explore the physical properties of our candidates, we use the diagnostics developed to identify BCDs in large galaxy samples, to see whether our candidates conform to the properties of known XMP galaxies. Following Sánchez Almeida et al. (2008), which condense the criteria by Gil de Paz et al. (2003) and Malmberg (2005), BCDs can be observationally characterized by,

(a) $\quad \mu_{g}-\mu_{r} \leq 0.43 \mathrm{mag} \operatorname{arcsec}^{-2}$,

(b) $\mu_{g}<21.83-0.47\left(\mu_{g}-\mu_{r}\right) \operatorname{mag} \operatorname{arcsec}^{-2}$,

(c) $\quad M_{g}>-19.12+1.72\left(M_{g}-M_{r}\right) \mathrm{mag}$,

(d) $\quad W_{\mathrm{H} \alpha}>50 \AA$,

(e) $12+\log (\mathrm{O} / \mathrm{H})<8.2(\equiv 1 / 3 \odot)$,

(f) no AGN,

(g) no bright galaxy within $10 R_{50}$.

The various new symbols have their usual meaning $-\mu_{g}$ and $\mu_{r}$ stand for the mean surface brightness in the SDSS filters $g$ and $r, M_{g}$ and $M_{r}$ represent the absolute magnitudes in these two filters, and $R_{50}$ is the radius containing $50 \%$ of the galaxy light. The criteria above assure that (a) BCDs are blue, (b) they have high surface brightness, (c) they are dwarf, (d) they have a large star-formation rate, (e) they are metal poor, (f) they are not confused with active galactic nuclei (AGN), and (g) they have no close companions. Figure 4 shows various scatter plots relating the physical parameters involved in BCD characterization. The required colors and sizes have been taken directly from the SDSS/DR7 database using Petrosian magnitudes (Stoughton et al. 2002a; Abazajian et al. 2009). The color-magnitude plot, Fig. 4b, shows that all our XMP candidates are dwarf - the dashed line represents constraint (c). Their $\mathrm{H} \alpha$ equivalent width exceeds the required threshold (constraint $\mathrm{d}$ ), and the galaxies reside in a region of the BPT diagram (after Baldwin et al. 1981) discarding the AGN nature the dashed line in Fig. 4 d divide AGN activity and star-forming activity as worked out by Kauffmann et al. (2003). The XMP candidates obviously fulfill criterion (e) (see Fig. 3), and they are also blue-enough, staying below the horizontal dashed line in Fig. 4a corresponding to criterion (a). As far as the compactness criterion (b) is concerned, most galaxies comply with it, being to the left of the slanted dashed line in Fig. 4a. Four of them do not - they are represented as asterisks in Fig. 4a, as well as in the remaining panels of the figure. The low surface brightness of these galaxies is to some extent apparent. Visual inspection of their SDSS composite-color images show two of them to present a marked cometary shape or double nucleus, and the SDSS reduction pipeline has probably overestimated the apparent size of those galaxies. 
Spurred by the unusual shape of these non-BCD galaxies, we carry out an eyeball inspection of all the 32 targets. Many of them turned out to have distorted morphologies, often with cometary shape, knots and/or chains of knots. Only 8 appear as a single knot without obvious substructure. The results of this crude morphological classification are given in Table 1. Figure 5 includes SDSS mugshots of all XMP candidates not marked as single knot in Table. 1. Those galaxies tagged with a question mark in Table 1 represent ambiguous cases where the cometary shape is less obvious, however, even in theses cases the images show elongated asymmetrical substructure (e.g., \# 29).

The color magnitude diagram in Fig. 4b deserves a separate comment. First, it shows how several XMP candidates are extremely blue, reaching up to $g-r \lesssim-1$. These extreme colors are rare, but they have been quoted in connection with luminous star-forming galaxies (e.g., Izotov et al. 2011), and with blue compact dwarfs (e.g., Sánchez Almeida et al. 2008). Second, the figure suggests a trend that contrasts with usual behavior where brighter galaxies tend to be redder (e.g., Blanton \& Moustakas 2009) - the brighter the XMP candidate the bluer. This unusual trend cannot be ascribed to photometric errors (the error bars provided by SDSS are included in Fig. 4b). The behavior remains independently of the type of magnitude (Fig. 4b uses Petrosian magnitudes, but we also tried the other magnitudes provided by SDSS; see Stoughton et al. 2002b). Moreover, the trend disappears when colors other than $g-r$ are used. This unusual trend calls for an explanation but, so far, we can only offer conjectures. It may be a random fluctuation, since only a handful of galaxies define the trend. Alternatively, it may be due to a subtle effect on the integrated colors of the large emission lines that dominate the spectrum of these galaxies.

In short, all but four of the 32 candidates fulfill the criteria to be BCDs, and 24 of them show cometary or knotted shape. The fact that the XMP candidates have these properties is by no means trivial. We have selected our sample according to the form of their spectra in an narrow spectral window around $\mathrm{H} \alpha$, and this narrow bit of spectrum turns out to determine many global properties of the galaxy such as color, compactness, star formation rate, and even morphology.

\section{Search for XMP galaxies in the literature}


Table 2. XMP targets found in the literature.

\begin{tabular}{|c|c|c|c|c|c|c|}
\hline Name $^{\mathrm{a}}$ & RA & DEC & $g$ & SpecObjID $^{\mathrm{b}}$ & $12+\log (\mathrm{O} / \mathrm{H})$ & Comment $^{\mathrm{c}}$ \\
\hline UGC 12894 & 000022 & +392944 & & & 7.64 & van Zee \& Haynes (2006) \\
\hline $\mathrm{J} 0004+0025$ & 000422 & +002536 & 19.4 & 433417380224303104 & 7.37 & Guseva et al. (2009) \\
\hline J0014-0044 & 001429 & -004444 & 18.7 & 306753510077104128 & 7.63 & Guseva et al. (2009) \\
\hline J0015+0104 & 001521 & +010437 & 18.3 & 433983718529433600 & 7.07 & Guseva et al. (2009) \\
\hline $\mathrm{J} 0016+0108$ & 001628 & +010802 & 18.9 & 193598873954942976 & 7.53 & Guseva et al. (2009) \\
\hline HS $0017+1055$ & 002021 & +111221 & & & 7.63 & Pustilnik \& Martin (2007) \\
\hline J0029-0108 & 002905 & -010826 & 19.2 & 434546692474273792 & 7.35 & Guseva et al. (2009) \\
\hline J0029-0025 & 002949 & -002540 & 20.4 & 434546692394582016 & 7.29 & Guseva et al. (2009) \\
\hline ESO 473- G024 & 003122 & -224557 & & & 7.45 & Skillman et al. (2003) \\
\hline Andromeda IV & 004232 & +403419 & & & 7.49 & Pustilnik et al. (2008) \\
\hline J0057-0022 & 005713 & -002158 & 19.1 & 305062972235972608 & 7.60 & Guseva et al. (2009) \\
\hline IC 1613 & 010448 & +020704 & & & 7.64 & Nagao et al. (2006) \\
\hline J0107+0001 & 010751 & +000128 & 19.4 & 422158628955357184 & 7.23 & Guseva et al. (2009) \\
\hline AM 0106-382 & 010822 & -381234 & & & 7.56 & Lee et al. (2003) \\
\hline $\mathrm{J} 0113+0052$ & 011340 & +005239 & 20.1 & 422158629852938240 & 7.24 & Izotov \& Thuan (2007) \\
\hline J0119-0935 & 011914 & -093546 & 19.5 & 185997585616470016 & 7.31 & Ekta \& Chengalur (2010b) \\
\hline HS $0122+0743^{\star}$ & 012534 & +075924 & 15.7 & 655785970125242368 & 7.60 & Izotov \& Thuan (2004b) \\
\hline J0126-0038 & 012646 & -003839 & 18.4 & 422724752210132992 & 7.51 & Guseva et al. (2009) \\
\hline $\mathrm{J} 0133+1342$ & 013353 & +134209 & 18.1 & 120131172889001984 & 7.56 & Papaderos et al. (2008) \\
\hline J0135-0023 & 013544 & -002317 & 18.9 & 303656125474013184 & 7.38 & Guseva et al. (2009) \\
\hline UGCA20 & 014315 & +195832 & 18.0 & & 7.60 & KÖ, van Zee \& Havnes (2006) \\
\hline UM133 & 014442 & +045342 & 15.4 & & 7.63 & KÖ, Kniazev et al. (2001) \\
\hline HKK97L14 & 020010 & +284953 & & & 7.56 & van Zee \& Havnes (2006) \\
\hline J0204-1009 & 020426 & -100935 & 17.1 & 187686313107914752 & 7.36 & Izotov \& Thuan (2007) \\
\hline J0205-0949 & 020549 & -094918 & 15.3 & 187967849057222656 & 7.61 & Kniazev et al. (2003) \\
\hline J0216+0115 & 021629 & +011521 & 17.4 & 424413702440091648 & 7.44 & Guseva et al. (2009) \\
\hline 096632 & 025147 & -300632 & 16.3 & & 7.51 & Guseva et al. (2009) \\
\hline J0254+0035 & 025429 & +003550 & 19.8 & 425817950054776832 & 7.28 & Izotov \& Thuan (2007) \\
\hline J0301-0059 & 030126 & -005926 & 21.5 & 300559785177120768 & 7.64 & Guseva et al. (2009) \\
\hline J0301-0052 & 030149 & -005257 & 18.8 & 300559785005154304 & 7.52 & Izotov \& Thuan (2007) \\
\hline J0303-0109* & 030331 & -010947 & 19.8 & 225967511814799360 & 7.22 & Guseva et al. (2009) \\
\hline J0313+0010 & 031302 & +001040 & 18.9 & 226248880952442880 & 7.44 & Izotov \& Thuan (2007) \\
\hline J0315-0024 & 031600 & -002426 & 20.2 & 426661932058017792 & 7.41 & Guseva et al. (2009) \\
\hline UGC2684 & 032024 & +171745 & 22.8 & & 7.60 & KÖ, van Zee \& Haynes (2006) \\
\hline SBS0335-052W & 033738 & -050237 & 19.0 & & 7.11 & KÖ, Thuan \& Izotov (2005) \\
\hline SBS0335-052E & 033744 & -050240 & 16.3 & & 7.31 & KÖ, Thuan \& Izotov (2005) \\
\hline J0338+0013 & 033812 & +001313 & 24.4 & 227094714526990336 & 7.64 & Guseva et al. (2009) \\
\hline J0341-0026 & 034118 & -002628 & 18.8 & 325892648806121472 & 7.26 & Guseva et al. (2009) \\
\hline ESO 358- G 060 & 034512 & -353415 & & & 7.26 & Lee et al. (2003) \\
\hline G0405-3648 & 040519 & -364849 & & & 7.25 & Guseva et al. (2009) \\
\hline J0519+0007 & 051903 & +000729 & 18.4 & & 7.44 & Guseva et al. (2009) \\
\hline To0618-402 & 062002 & -401809 & & & 7.56 & KÖ, Masegosa et al. (1994) \\
\hline ESO489-G56 & 062617 & -261556 & 15.6 & & 7.49 & KÖ, Roennback \& Bergvall (1995) \\
\hline $\mathrm{J} 0808+1728^{\star}$ & 080841 & +172856 & 19.2 & 585978593608204288 & 7.48 & Brown et al. (2008) \\
\hline J0812+4836 & 081239 & +483646 & 16.0 & 124071834843873280 & 7.28 & Izotov \& Thuan (2007) \\
\hline UGC 4305 & 081905 & +704312 & & & 7.65 & Nagao et al. (2006) \\
\hline
\end{tabular}




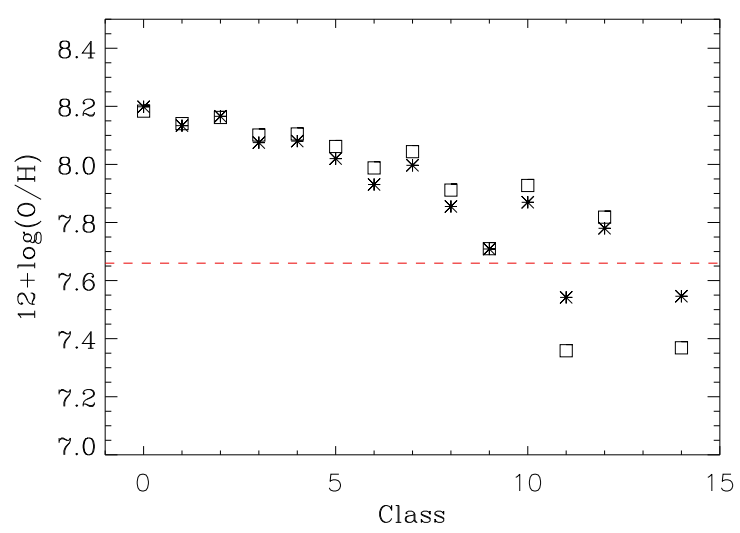

Fig. 3.- Oxygen abundance of the various classes as inferred using the N2 method on the template spectra corresponding to each one the classes. The two types of symbols correspond to the two slightly different calibrations in Pettini \& Pagel (2004) - asterisks for equation (3) and boxes for equation (44). The horizontal dashed line represents one-tenth of the solar metallicity (Asplund 2005). 

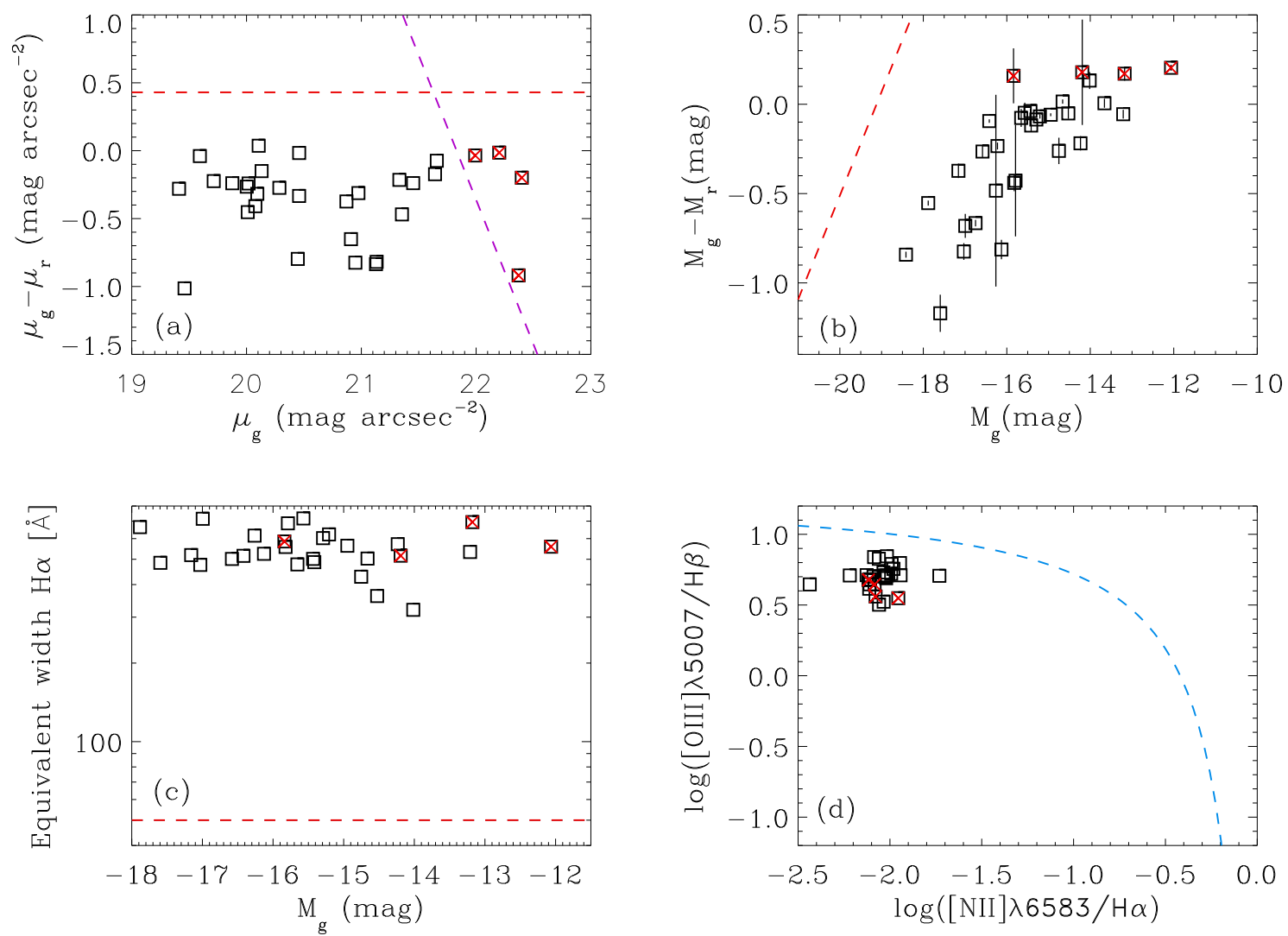

Fig. 4. - Diagnostic plots to characterize the XMP galaxies resulting from the search. (a) Color $\mu_{g}-\mu_{r}$ vs surface brightness in the $g$ filter $\mu_{g}$. Only 4 out of the 32 galaxies have a surface brightness beyond the slanted line that bounds BCD galaxies. These four outliers are marked with times symbols (in red) in all the plots. (b) Color $M_{g}-M_{r}$ vs absolute magnitude $M_{g}$ in the $g$ filter. All the galaxies are consistent with being BCDs. The error bars have been taken directly from SDSS. (c) $\mathrm{H} \alpha$ equivalent width vs absolute magnitudes. The starformation rate inferred from these equivalent widths exceeds in all cases the BCD threshold (the dashed line). (d) BPT diagram showing all the targets to be starforming galaxies as opposed to AGNs (separated by the dashed curve worked out by Kauffmann et al. 2003). 

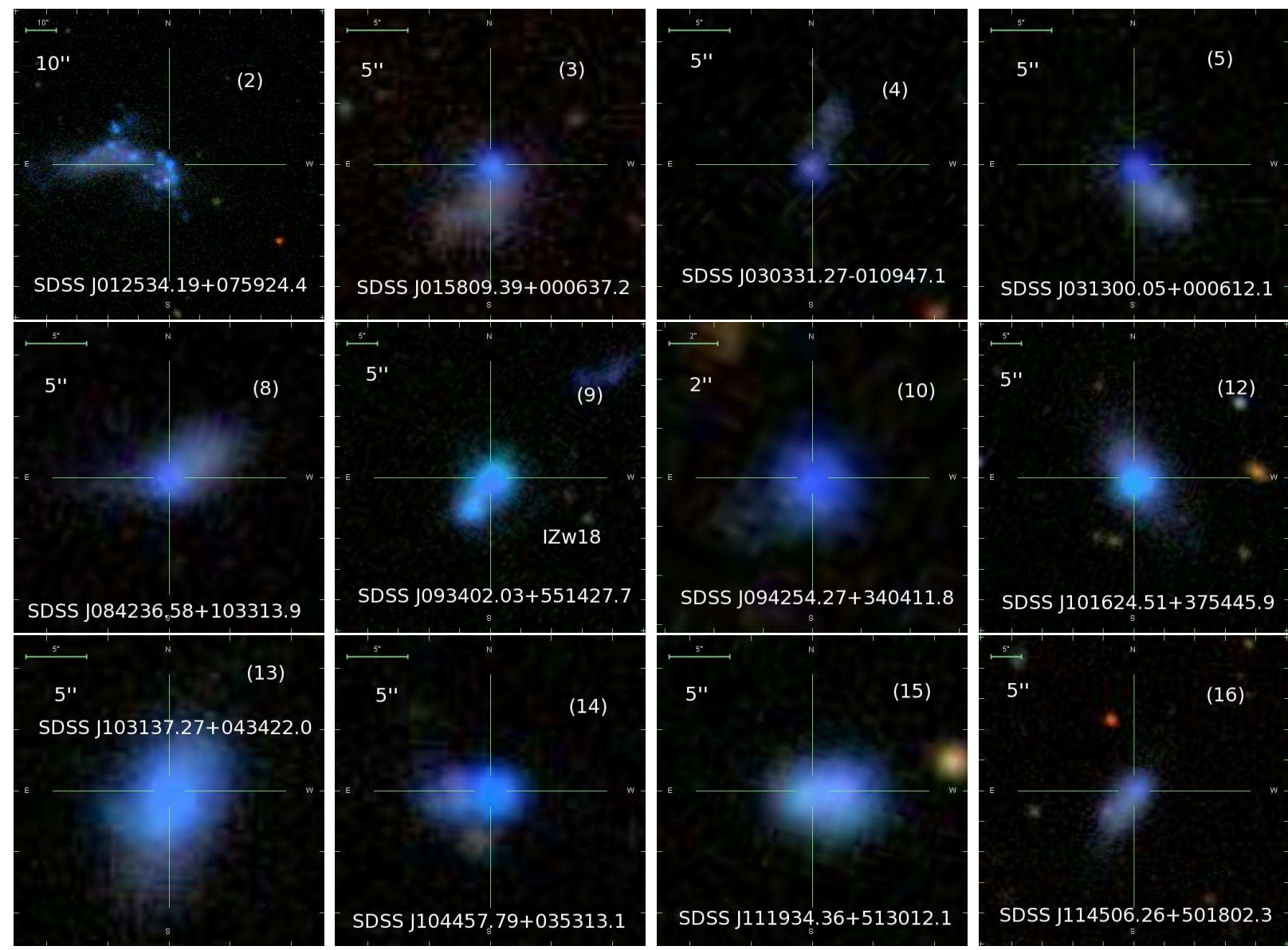

Fig. 5.- SDSS mugshots of all XMP candidates not marked as single knot in Table. 1, They are cometary (e.g., \# 5), knotted-cometary (e.g., \# 2 ), or doubtful (e.g., \# 13). The numbers correspond to the index in Table. 1, whereas the scales on the top left corner of the panels represent 2, 5 or 10 arcsec, as indicated by the insets. The figure continues in Fig. 5 , 

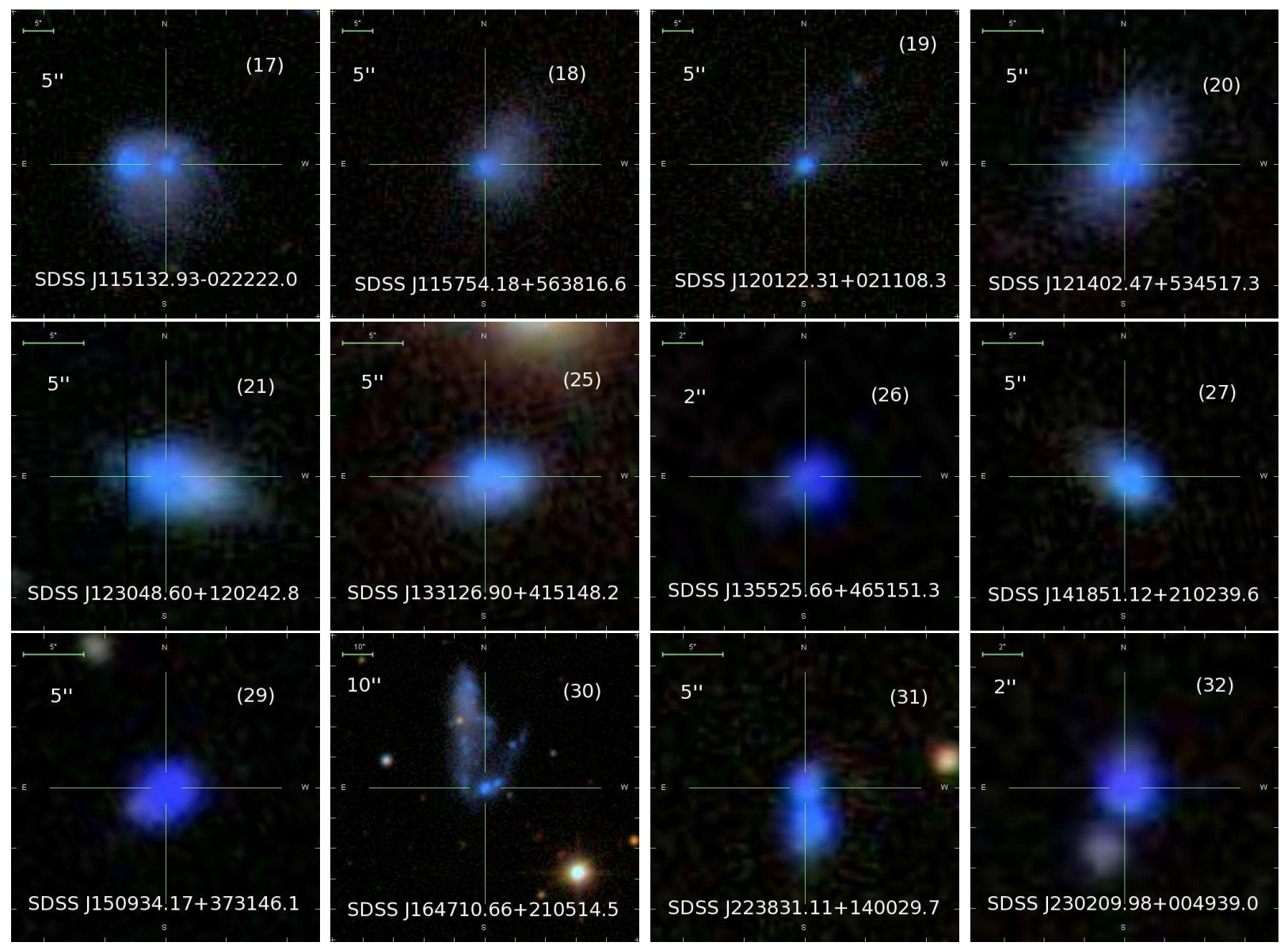

Fig. 5.- Continuation of Fig. 5 . 
Table 2-Continued

\begin{tabular}{|c|c|c|c|c|c|c|}
\hline $\mathrm{Name}^{\mathrm{a}}$ & RA & $\mathrm{DEC}$ & $g$ & SpecObjID ${ }^{b}$ & $12+\log (\mathrm{O} / \mathrm{H})$ & Comment $^{\mathrm{c}}$ \\
\hline HSO $022+03542$ & 082555 & +353231 & 17.8 & & 7.35 & KÖ, Kniazev et al. (2000) \\
\hline DDO53 & 083407 & +661054 & 20.3 & & 7.62 & KÖ, van Zee et al. (2006) \\
\hline UGC4483 & 083703 & +694631 & 15.1 & & 7.58 & KÖ, Izotov \& Thuan (2002) \\
\hline HS0837+4717 & 084030 & +470710 & 17.6 & & 7.64 & Pustilnik et al. (2004) \\
\hline HS $0846+3522$ & 084940 & +351139 & 18.2 & & 7.65 & Pustilnik \& Martin (2007) \\
\hline J0859+3923 & 085947 & +392306 & 17.2 & & 7.57 & Izotov \& Thuan (2007) \\
\hline $\mathrm{J} 0910+0711$ & 091029 & +071118 & 16.9 & 336307481519063040 & 7.63 & Ekta \& Chengalur (2010b) \\
\hline J0911+3135 & 091159 & +313536 & 17.8 & 448054218540974080 & 7.51 & Izotov \& Thuan (2007) \\
\hline J0926+3343 & 092609 & +334304 & 17.8 & 448617233443192832 & 7.12 & Pustilnik et al. (2010) \\
\hline $\mathrm{IZw} 18^{\star}$ & 093402 & +551425 & 16.4 & 156443095175528448 & 7.17 & KÖ, Thuan \& Izotov (2005) \\
\hline J0940+2935 & 094013 & +293530 & 16.5 & 546853820680896512 & 7.65 & Izotov \& Thuan (2007) \\
\hline CGCG 007-025 & 094402 & -003832 & 16.0 & 75094093385957376 & 7.65 & Guseva et al. (2007) \\
\hline SBS940+544 & 094417 & +541134 & 19.1 & & 7.46 & KÖ, Guseva et al. (2001) \\
\hline CS 0953-174 & 095500 & -170000 & & & 7.58 & KÖ, Masegosa et al. (1994) \\
\hline J0956+2849 & 095646 & +284944 & 15.9 & 548261264221011968 & 7.13 & Izotov \& Thuan (2007) \\
\hline LeoA & 095926 & +304447 & 19.0 & & 7.30 & KÖ, van Zee \& Havnes (2006) \\
\hline Sextans B & 100000 & +051956 & 20.5 & & 7.53 & Lee et al. (2006) \\
\hline Sextans A & 101100 & -044134 & & & 7.54 & KÖ, Kniazev et al. (2005) \\
\hline KUG $1013+381^{\star}$ & 101624 & +375444 & 15.9 & 401892408779866112 & 7.58 & KÖ, Kniazev \& Pustil'Nik (1998) \\
\hline SDSS J1025+1402 & 102530 & +140207 & 20.4 & 491964741116231680 & 7.36 & Izotov et al. (2007a) \\
\hline UGCA 211 & 102702 & +561614 & 16.2 & & 7.56 & Cairós et al. (2010) \\
\hline $\mathrm{J} 1031+0434^{\star}$ & 103137 & +043422 & 16.2 & 162635977557278720 & 7.70 & Izotov et al. (2007a) \\
\hline HS $1033+4757$ & 103625 & +474152 & 17.5 & 271004930616066048 & 7.65 & Pustilnik \& Martin (2007) \\
\hline $\mathrm{J} 1044+0353^{\star}$ & 104458 & +035313 & 17.5 & 162917331083722752 & 7.44 & Papaderos et al. (2008) \\
\hline HS $1059+3934$ & 110210 & +391845 & 17.9 & & 7.62 & Pustilnik \& Martin (2007) \\
\hline $\mathrm{J} 1105+6022$ & 110554 & +602229 & 16.4 & 218086199200317440 & 7.64 & Kniazev et al. (2003) \\
\hline $\mathrm{J} 1119+5130^{\star}$ & 111934 & +513012 & 16.9 & 247359886311030784 & 7.51 & KÖ, Kniazev et al. (2003) \\
\hline $\mathrm{J} 1121+0324$ & 112153 & +032421 & 18.1 & 235538034580258816 & 7.64 & Kniazev et al. (2003) \\
\hline UGC 6456 & 112800 & +785939 & & & 7.35 & Nagao et al. (2006) \\
\hline SBS1129+576 & 113202 & +572246 & 16.7 & & 7.36 & Guseva et al. (2003c) \\
\hline $\mathrm{J} 1151-0222^{\star}$ & 115132 & -022222 & 16.8 & 93111671593107456 & 7.78 & Nagao et al. (2006) \\
\hline $\mathrm{J} 1201+0211^{\star}$ & 120122 & +021108 & 17.6 & 145464500043120640 & 7.49 & Papaderos et al. (2008) \\
\hline SBS1159+545 & 120202 & +541550 & 18.7 & & 7.41 & KÖ, Nava et al. (2006) \\
\hline SBS $1211+540^{\star}$ & 121402 & +534517 & 17.4 & 287049377199947776 & 7.64 & Nagao et al. (2006) \\
\hline $\mathrm{J} 1215+5223$ & 121547 & +522314 & 15.2 & 248767590061572096 & 7.43 & Kniazev et al. (2003) \\
\hline Tol1214-277 & $1217 \quad 17$ & -280233 & & & 7.55 & KÖ, Izotov et al. (2004) \\
\hline VCC 0428 & 122040 & +135322 & 17.0 & 497314452257898496 & 7.64 & Vílchez \& Iglesias-Páramo (2003) \\
\hline HS $1222+3741$ & 122437 & +372437 & 17.9 & 564023911881113600 & 7.64 & Popescu \& Hopp $(2000)^{*}$ \\
\hline Tol65 & 122547 & -361401 & 17.5 & & 7.54 & KÖ, Izotov et al. (2004) \\
\hline $\mathrm{J} 1230+1202^{\star}$ & 123049 & +120243 & 16.7 & 454810434122285056 & 7.73 & KÖ, Pustilnik et al. (2002) \\
\hline KISSR 85 & 123718 & +291455 & 19.9 & & 7.61 & Lee et al. (2004) \\
\hline UGCA 292 & 123840 & +324601 & 18.9 & & 7.28 & Pilyugin (2001) \\
\hline HS $1236+3937$ & 123920 & +392105 & 18.5 & & 7.47 & Popescu \& Hopp $(2000)^{*}$ \\
\hline $\mathrm{J} 1239+1456$ & 123945 & +145613 & 19.8 & & 7.65 & Brown et al. (2008) \\
\hline SBS $1249+493$ & 125152 & +490328 & 18.0 & & 7.64 & Nava et al. (2006) \\
\hline $\mathrm{J} 1255-0213^{\star}$ & 125526 & -021334 & 19.1 & 95080395053203456 & 7.83 & Yin et al. (2007) \\
\hline
\end{tabular}


In order to put our search into context, we carried out a careful revision of the literature to select all the nearby galaxies with metallicity reported to be one-tenth solar or less. $(12+\log (\mathrm{O} / \mathrm{H})<7.65)$. Those are listed in Table 2, We start off from Table 3 in Kunth \& Östlin (2000), who compile all the galaxies found in the literature prior to year 2000. It comprises 31 targets, from which $\mathbf{5}$ were discarded because their metallicity has been revised upwards. Then we cover the last ten years by checking cross-references existing in recent known papers. We begin with the work by Guseva et al. (2009), that analyzes 44 targets selected in SDSS/DR6. The spectra used in the analysis are not spectra from SDSS but obtained elsewhere. We choose from Guseva et al. (2009) all the galaxies with oxygen abundance below the threshold. Then, using ADS3, we revised all papers cited in Guseva et al. (2009) and published after Kunth \& Östlin (2000). Based on their title and abstract, we examined those papers dealing with galaxy metallicity, separating the appropriate XMP galaxies. High redshift targets were not included (e.g., those in Lilly et al. 2003). The procedure was repeated with all the papers containing XMP galaxies, until no new reference earlier than Kunth \& Östlin (2000) was found. In addition, our recursive searching strategy was repeated with all the papers citing Guseva et al. (2009). For example, Izotov \& Thuan (2007) is cited by Guseva et al. (2009) and provides 13 targets from SDSS/DR5. Papaderos et al. (2008) study spectroscopically and morphologically 7 galaxies identified in SDSS/DR4 and 6dFGS (six-degree field galaxy survey, Jones et al. 2004). Three galaxies are exhaustively analyzed in three separate papers by Guseva et al. (2003a, b. c), and are also included in Table 2, Two additional galaxies are from the study by Izotov et al. (2006a). Through this step-by-step search procedure, we revised all the papers that seems to be relevant, and a number of them rendered one or several additional XMP galaxies for Table 2 (Popescu \& Hopp 2000; Kniazev et al. 2000, 2001; Guseva et al. 2001; Pilvugin 2001; Hidalgo-Gámez \& Olofsson 2002; Izotov \& Thuan 2002; Lee et al. 2003; Guseva et al. 2003c; Skillman et al. 2003; Vílchez \& Iglesias-Páramo 2003; Kniazev et al. 2003; Pustilnik et al. 2004; Izotov et al. 2004; Izotov \& Thuan 2004b; Thuan \& Izotov 2005; Kniazev et al. 2005; Papaderos et al. 2006; Nagao et al. 2006; van Zee \& Havnes 2006; van Zee et al. 2006; Nava et al. 2006; Lee et al. 2006; Pustilnik et al. 2006; Guseva et al. 2007; Pustilnik \& Martin 2007; Izotov \& Thuan 2007; Izotov et al. 2007a; Papaderos et al. 2008; Pustilnik et al. 2008; Brown et al. 2008; Guseva et al. 2009; Pustilnik et al. 2010; Ekta \& Chengalur 2010b; Cairós et al. 2010). Finally, the galaxies in Table 1 were inspected individually in NED4, to assure that they are included in our bibliographic search even when the estimated metallicity exceeds the imposed threshold. Table 2 contains all galaxies we found in the literature through this search. It

\footnotetext{
${ }^{3}$ The digital library NASA Astrophysical Data System http://www.adsabs.harvard.edu/

${ }^{4}$ NASA Extragalactic Data base http://nedwww.ipac.caltech.edu/.
} 
Table 2-Continued

\begin{tabular}{|c|c|c|c|c|c|c|}
\hline $\mathrm{Name}^{\mathrm{a}}$ & RA & DEC & $g$ & SpecObjID ${ }^{b}$ & $12+\log (\mathrm{O} / \mathrm{H})$ & Comment $^{\mathrm{c}}$ \\
\hline Gr8 & 125840 & +141303 & 17.9 & & 7.65 & KÖ, van Zee \& Havnes (2006) \\
\hline KISSR 1490 & 131316 & +440230 & 19.0 & & 7.56 & Lee et al. (2004) \\
\hline DDO 167 & 131323 & +461922 & & & 7.20 & Hidalgo-Gámez \& Olofsson (2002) \\
\hline HS $1319+3224$ & 132120 & +320825 & 18.6 & & 7.59 & Popescu \& Hopp (2000) \\
\hline $\mathrm{J} 1323-0132^{\star}$ & 132347 & -013252 & 18.2 & 96204976459612160 & 7.78 & Izotov et al. (2007a) \\
\hline $\mathrm{J} 1331+4151^{\star}$ & 133127 & +415148 & 17.1 & 412307391565004800 & 7.75 & Izotov et al. (2007b) \\
\hline ESO577-G27 & 134247 & -193454 & & & 7.57 & KÖ, Roennback \& Bergvall (1995) \\
\hline $\mathrm{J} 1355+4651^{\star}$ & 135526 & +465151 & 19.3 & 361921789577658368 & 7.63 & Pustilnik et al. (2005) \\
\hline J1414-0208 & 141454 & -020823 & 18.0 & 258056040799535104 & 7.28 & Papaderos et al. (2008) \\
\hline SBS1415+437 & 141701 & +433005 & 17.8 & & 7.43 & KÖ, Nava et al. (2006) \\
\hline $\mathrm{J} 1422+5145$ & 142251 & +514516 & 20.2 & & 7.41 & Brown et al. (2008) \\
\hline $\mathrm{J} 1423+2257^{\star}$ & 142343 & +225729 & 17.9 & 600334402043510784 & 7.72 & Izotov et al. (2007b) \\
\hline $\mathrm{J} 1441+2914$ & 144158 & +291434 & 20.1 & & 7.47 & Brown et al. (2008) \\
\hline HS1442+4250 & 144413 & +423744 & 15.9 & & 7.54 & Guseva et al. (2003b) \\
\hline $\mathrm{J} 1509+3731^{\star}$ & 150934 & +373146 & 17.3 & 394011865673367552 & 7.85 & Izotov et al. (2007b) \\
\hline KISSR 666 & 151542 & +290140 & 19.1 & & 7.53 & Nagao et al. (2006) \\
\hline KISSR 1013 & 161639 & +290333 & 18.2 & 396262437692637184 & 7.63 & Nava et al. (2006) \\
\hline $\mathrm{J} 1644+2734$ & 164403 & +273405 & 17.7 & 475922385527111680 & 7.48 & Izotov et al. (2007a) \\
\hline $\mathrm{J} 1647+2105^{\star}$ & 164711 & +210515 & 17.3 & 442143986740625408 & 7.75 & Izotov et al. (2007b) \\
\hline $\mathrm{W} 1702+18$ & 170233 & +180306 & 18.4 & & 7.63 & Griffith et al. (2011) \\
\hline HS $1704+4332$ & 170545 & +432849 & 18.4 & & 7.55 & Pustilnik \& Martin (2007) \\
\hline SagDIG & 192959 & -174041 & & & 7.44 & KÖ, van Zee et al. (2006) \\
\hline $\mathrm{J} 2053+0039$ & 205313 & +003915 & 19.4 & 288175754707992576 & 7.33 & Guseva et al. (2009) \\
\hline $\mathrm{J} 2104-0035$ & 210455 & -003522 & 17.9 & 288457262211530752 & 7.05 & Guseva et al. (2009) \\
\hline $\mathrm{J} 2105+0032$ & 210509 & +003223 & 19.0 & 288457264749084672 & 7.42 & Guseva et al. (2009) \\
\hline $\mathrm{J} 2120-0058$ & 212026 & -005827 & 18.8 & 289300532823064576 & 7.65 & Guseva et al. (2009) \\
\hline HS2134+0400 & 213659 & +041404 & & & 7.44 & Pustilnik et al. (2006) \\
\hline $\mathrm{J} 2150+0033$ & 215032 & +003305 & 19.3 & 414839880762261504 & 7.60 & Guseva et al. (2009) \\
\hline ESO146-G14 & 221300 & -620403 & & & 7.59 & KÖ, Roennback \& Bergvall (1995) \\
\hline $2 \mathrm{dF} 171716$ & 221326 & -252643 & & & 7.54 & Papaderos et al. (2006) \\
\hline PHL293B & 223037 & -000637 & 17.2 & & 7.62 & Guseva et al. (2009) \\
\hline $2 \mathrm{dF} 115901$ & 223702 & -285241 & & & 7.57 & Papaderos et al. (2006) \\
\hline $\mathrm{J} 2238+1400^{\star}$ & 223831 & +140030 & 19.0 & 533060792673632256 & 7.45 & Guseva et al. (2009) \\
\hline $\mathrm{J} 2250+0000$ & 225059 & +000033 & 19.8 & 190501187865280512 & 7.61 & Izotov et al. (2007a) \\
\hline $\mathrm{J} 2259+1413$ & 225901 & +141343 & 19.1 & & 7.37 & Brown et al. (2008) \\
\hline $\mathrm{J} 2302+0049^{\star}$ & 230210 & +004939 & 18.8 & 190784502518251520 & 7.71 & Guseva et al. (2009) \\
\hline J2354-0005 & 235437 & -000502 & 18.7 & 307596757485748224 & 7.35 & Guseva et al. (2009) \\
\hline
\end{tabular}

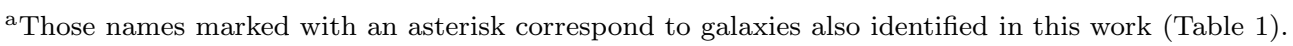

${ }^{\mathrm{b}}$ Unique identifier of the galaxy spectrum in SDSS/DR7.

${ }^{\mathrm{c}}$ Reference to the work where the oxygen abundance was obtained, plus the KÖ flag when the galaxy belongs to the compilation by Kunth \& Östlin (2000). 
includes only 129 galaxies, proving that the XMP targets are really rare objects. The table includes name, coordinates, SDSS SpecObjID (when available), the reference for the oxygen metallicity, as well as a tag to identify galaxies included in the work by Kunth \& Östlin (2000) used as reference. The match between Tables 1 and 2 renders 21 objects, i.e., only 21 of the 32 candidates had been previously identified as XMP galaxies. They are marked in the tables with asterisks. The remaining 11 objects are new. The overlapping between the two tables allows us to confirm the low metallicity of the XMP candidates selected using state-of-the-art metallicity determinations. The oxygen abundance in Table 2 are based on self-consistently determined electron temperatures. Assuming the twenty one known objects to be representative of the full set, then mean metallicity turns out to be $12+\log (\mathrm{O} / \mathrm{H})=7.61 \pm 0.19$, with the error bar accounting for the standard deviation.

Figure 6] shows the spatial distribution of the low metallicity targets, i.e., the $\mathbf{1 2 9}$ galaxies obtained from the literature plus the targets found in this work. Note that most works have focused the search in very localized areas on the sky. For example, the targets by Guseva et al. (2009) are clustered around the equator, even though the search is based on SDSS/DR6 which has a much broader scope. Our systematic search, however, has selected targets spread throughout the SDSS/DR7 field of view, that covers a significant part of the sky $(\sim 20 \%)$.

Figure 7 shows diagnostic plots like Fig. 4 but for the galaxies in the literature with spectra in SDSS. Assuming this subset to be representative of the full family, it shows several systematic differences with respect to the targets we have selected. The main one has to do with the ratio between $[\mathrm{NII}] \lambda 6583$ and $\mathrm{H} \alpha$, which is smaller than those characterizing our targets (cf. Fig. 4 $\mathrm{d}$, and Fig. 7 $\mathrm{d}$ ). Because of this difference, many of the XMP found in the literature would have escaped our search, which pick out classes with extreme contrast between $[\mathrm{NII}] \lambda 6583$ and $\mathrm{H} \alpha$. We do not have a final explanation for the difference, but we can offer two conjectures. Our search may yield galaxies in the low metallicity end of the XMP family, i.e., even more extreme than those existing in the literature and, consequently, even more metal poor than our estimates based on the semi-empirical calibrations by Pettini \& Pagel (2004). Alternatively, our search may be missing a fraction of the XMP galaxies, where the difference between the [NII] lines and $\mathrm{H} \alpha$ is not so extreme. The two possibilities explain other significant differences between our set and the targets from the literature. Our galaxies have larger surface brightness than those of the XMP galaxies in the literature (cf. Fig. $4 \mathrm{a}$ and Fig. $7 \mathrm{a}$ ). They have larger $\mathrm{H} \alpha$ equivalent widths as well. The surface brightness difference is attributable to the trend for the most metal poor galaxies to be BCDs (see $\S 3$ ). Alternatively, the physical conditions in the HII regions of the BCDs may differ systematically from other galaxies so that the same low metallicities render a particularly small ratio between $[\mathrm{NII}] \lambda 6583$ and $\mathrm{H} \alpha$ in BCDs. In order to sort out the two 


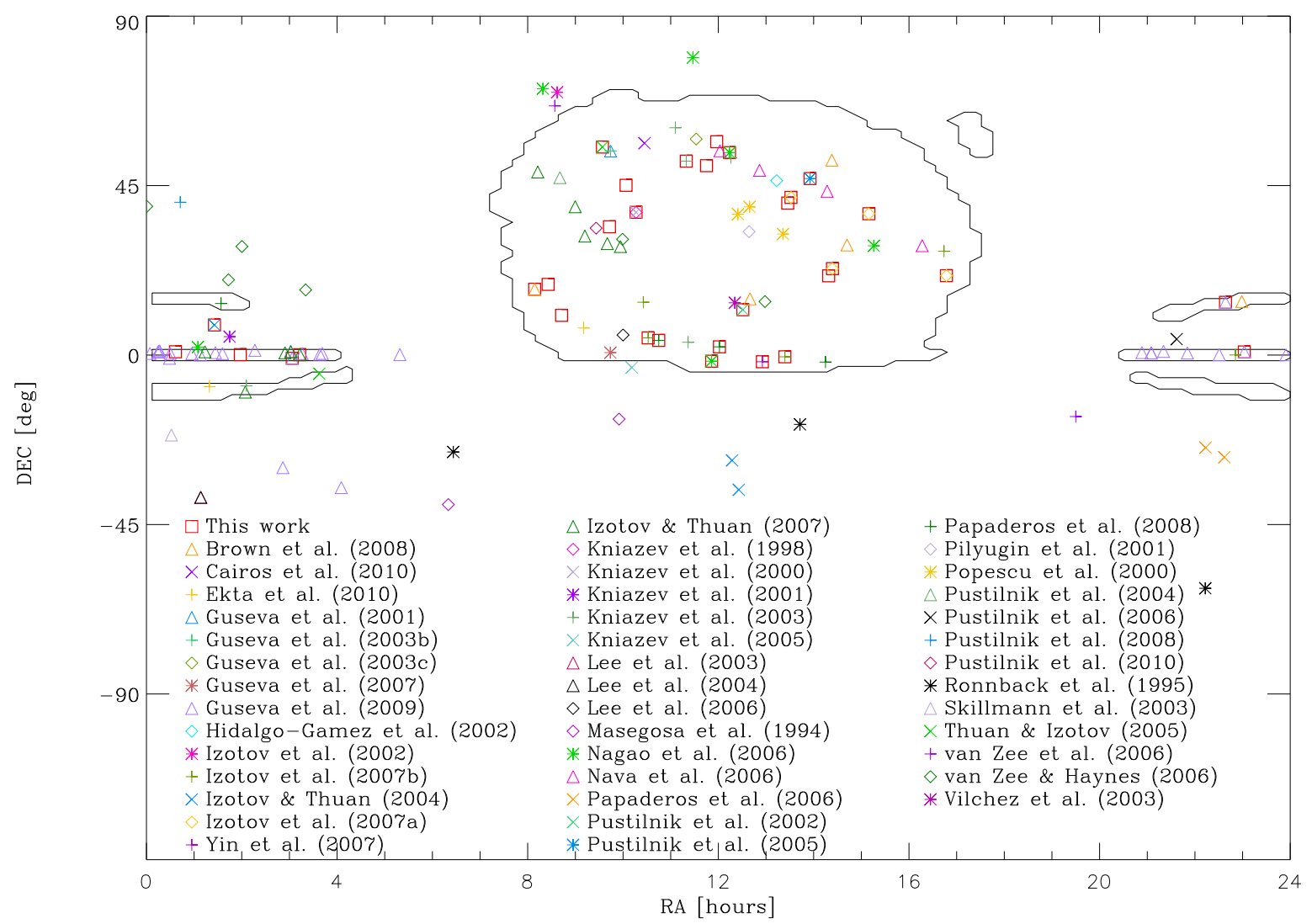

Fig. 6. - Spatial distribution of XMP galaxies, both of the $\mathbf{1 2 9}$ found in the literature, plus the 32 targets obtained in our systematic search through SDSS/DR7. Twenty one of them coincide. Different symbols and colors represent different sources as coded in the inset. In particular, the square symbols correspond to the galaxies found in our search. The contours represent the sky coverage of the spectroscopic sample of SDSS/DR7. Note that one of our targets is outside the main spectroscopic sample of SDSS/DR7 (i.e., it lies outside the contours). As usual, RA and DEC stand for right ascension and declination. 
possibilities one would have to determine the metallicities of our targets in a self-consistent way measuring electron temperatures and excitations (as in, e.g., Stasińska 2004; Izotov et al. $2006 \mathrm{~b})$. Such a detailed analysis clearly goes beyond the scope of the paper, and it is planned for following up work. Unfortunately, the twenty one candidates that were already known to be XMP do not clarify the situation. Some have particularly low metallicities, but not all of them (see the names with asterisks in Table 2).

\section{Number density of XMP galaxies in the local universe}

One of the advantages of carrying out a systematic search in SDSS/DR7 is starting from a magnitude limited sample. The relatively simple bias produced by this condition can be corrected for and, thus, the selected XMP galaxies can be used to estimate the volume number density of these unusual objects in the local universe. Such estimate is described in the present section.

Assume the metallicity $X$ of all galaxies in SDSS/DR7 to be known. Then the number of galaxies per unit volume and metallicity is just

$$
n(X)=\frac{1}{\Delta X} \sum_{i} \frac{1}{V_{i}} \Pi\left(\frac{X_{i}-X}{\Delta X}\right),
$$

where the sum over $i$ includes all the galaxies in the sample, $\Delta X$ represents the bin size of the metallicity histogram, the symbol $\Pi$ stands for the rectangle function,

$$
\Pi(x)= \begin{cases}1 & |x|<1 / 2, \\ 0 & \text { elsewhere }\end{cases}
$$

and $V_{i}$ represents the maximum volume in which the $i$-th galaxy of the sample could be observed. Equation (5) represents the so-called $V_{\max }$ approximation by Schmidt (1968) used to determine luminosity function of galaxies (e.g., Takeuchi et al. 2000), which has been transformed to derive the number density of any other physical property of the galaxies (e.g., Sánchez Almeida et al. 2008, § 4). Assume the sample to be magnitude limited, so that all galaxies brighter than the apparent magnitude $m_{l i m}$ are included. (This is the way the spectroscopic sample of SDSS was defined and therefore it must be good approximation.) Then,

$$
V_{i}=\frac{d_{i}^{3}}{3} \Omega,
$$

with $\Omega$ the solid angle covered by the survey and $d_{i}$ the maximum distance at which the $i$-th galaxy can be observed,

$$
\log \left(d_{i} / 1 \mathrm{Mpc}\right)=\left(m_{\text {lim }}-M_{i}\right) / 5-5,
$$



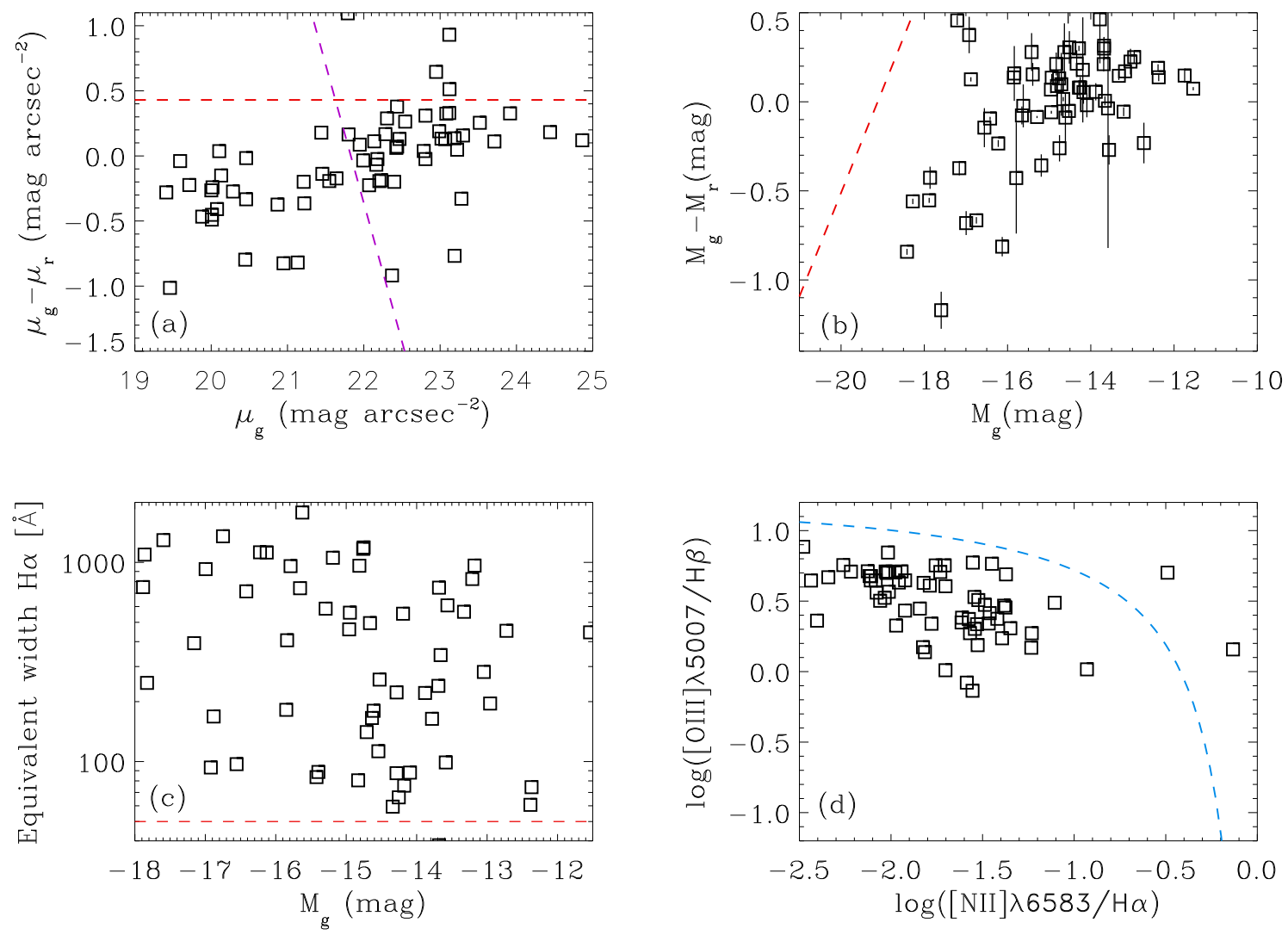

Fig. 7.- Similar to Fig. 4, but including previously known XMP galaxies with spectra in SDSS/DR7. (a) Color $\mu_{g}-\mu_{r}$ vs surface brightness in the $g$ filter $\mu_{g}$. Note that these targets have systematically lower surface brightness than the ones we find, and many of them cannot be considered BCDs according to the criteria used in $\S 3$, (b) Color $M_{g}-M_{r}$ vs absolute magnitude $M_{g}$ in the $g$ filter. The error bars have been taken directly from SDSS. (c) $\mathrm{H} \alpha$ equivalent width vs absolute magnitudes. (d) BPT diagram showing most of the targets to be starforming galaxies. The outlier comes from the sample of special targets with broad emission lines selected by Izotov et al. (2007a), where the integrated spectra are known to have a significant $\mathrm{SNa}$ and/or AGN contamination. In general, the ratio between [NII] $\lambda 6583$ and $\mathrm{H} \alpha$ is not as small as it is in our selection. 
which only depends on its absolute magnitude $M_{i}$. A few comments and caveats are in order before applying equation (5) to the dataset. First, SDSS is not truly magnitude limited. Some bright galaxies are not observed because of problems to pack the spectrograph fibers in crowded fields (see Stoughton et al. 2002a) and, as usual, very low surface brightness galaxies tend to be missed even if they are luminous (e.g., Blanton et al. 2005). None of these two problems seems to be of relevance for the XMP galaxies, which are both isolated and high surface brightness $\left(\S[\right.$ ] $)$. SDSS/DR7 sets $m_{\text {lim }}=17.8$ in $r$, but our selection also imposes a cut at redshift $\leq 0.25$ which, in principle, modifies the magnitude-limited character of the original sample. This question is of no concern, though. Our targets are dwarf galaxies, never reaching a luminosity sufficient to be detected beyond the redshift threshold. Finally, computing the galaxy metallicities using detailed chemical abundance analyses (as in, e.g., Stasińska 2004; Izotov et al. 2006b) goes beyond the scope of the paper. We estimate the metallicities using the strong line calibration of by Pettini \& Pagel (2004) described in $\S 2$, They are given in Table 1. Specifically, we employ the calibration in equation (4), which seems to be more appropriate for XMP galaxies where N2 -2 (N2 is the logarithm of ratio between the equivalent widths of the lines, as defined in equation [2]). The use of this approximation for the estimate of the metallicity implies that our estimates are only indicative.

The 32 XMP galaxies selected in $\S 2$, together with equations (44), (15), (6) , (7), and (8), render the local density of galaxies with (oxygen) metallicity less than, approximately, one tenth of the solar value, $12+\log (\mathrm{O} / \mathrm{H}) \leq 7.65$,

$$
\int_{-\infty}^{7.65} n(X) d X \simeq(1.32 \pm 0.23) \cdot 10^{-4} \mathrm{Mpc}^{-3}
$$

The error bar in the previous expression assumes the 32 targets to be drawn from a Poisson distribution. In terms of the total number of galaxies, XMP galaxies are just

$$
0.10 \pm 0.02 \%
$$

where the total number of galaxies in the local universe $\left(\simeq 0.13 \mathrm{Mpc}^{-3}\right)$ has been taken from the normalization of the luminosity functions by Blanton et al. (2003). These luminosity functions are also based on SDSS, and we use a Hubble constant of $70 \mathrm{~km} \mathrm{~s}^{-1} \mathrm{Mpc}^{-1}$ to revert their normalization. The error bar in equation (10) considers the dispersion quoted in equation (91) together with the spread of values among the different luminosity functions corresponding to the five SDSS color filter bandpasses.

Going a step further, we have considered all the galaxies with emission lines in SDSS/DR7 to estimate $n(X)$ in a broader range of metallicities. The SDSS/DR7 data reduction pipeline provides the equivalent widths of $[\mathrm{NII}] \lambda 6583$ and $\mathrm{H} \alpha$, which we use to estimate oxygen abundances. The distribution function $n(X)$ inferred from all these galaxies is shown in Fig. 8 a, 
the solid line. It has been computed using equation (5) with $\Delta X=0.2$, and considering only $\mathrm{N} 2 \leq-0.3(\equiv 12+\log (\mathrm{O} / \mathrm{H})<8.7)$, with this upper limit forced by the validity range of the calibration used to estimate abundances (see Pettini \& Pagel 2004). The horizontal bar in Fig. 8a corresponds to the density in equation (9), assuming the 32 XMP galaxies to spread one dex in metallicity. It is consistent with this other independent estimate inferred from the full distribution of SDSS/DR7 galaxies - if one integrates the histogram $n(X)$ in Fig. 8 a for galaxies with metallicities smaller than one-tenth solar $(12+\log (\mathrm{O} / \mathrm{H}) \leq 7.65)$, the volume number density of XMP galaxies turns out to be, $\int_{-\infty}^{7.65} n(X) d X \simeq 1.0 \cdot 10^{-4} \mathrm{Mpc}^{-3}$, which is close to the figure in equation (9). The integral of $n(X)$ in Fig. 8 a for all metallicities turns out to be $\simeq 5.2 \cdot 10^{-2} \mathrm{Mpc}^{-3}$, which is a factor two smaller than, but consistent with, the number density of local galaxies from Blanton et al. (2003) used in equation (10) for normalization. One have to keep in mind that the distribution in Fig. 8 a do not consider neither galaxies without emission lines nor galaxies with super-solar metallicities, which all together can easily account for the factor two difference.

XMP galaxies are dwarf and therefore they tend to be underrepresented in surveys. In order to illustrate the importance of such Malmquist bias, Fig. 8b contains the actual histogram of observed galaxies $N(X)$ from which $n(X)$ was derived. In the parlance of equation (5), it is given by

$$
N(X)=\sum_{i} \Pi\left(\frac{X_{i}-X}{\Delta X}\right) .
$$

Note how the low metallicity tail of $N(X)$ is depressed with respect to $n(X)$. This difference can be better appreciated in Fig. 8a, which also shows a scaled version of $N(X)$ forced to agree with $n(X)$ at the bin of highest metallicity. The figure shows how XMP galaxies are clearly lacking in the observation (the dashed line) as compared to their actual number densities (the solid line). We find than only $0.01 \%$ of the observed galaxies with emission lines are XMP, whereas they represent $0.2 \%$ considering galaxies in a fixed volume.

Obviously, the above estimates assume the XMP galaxy set to be complete. However, we cannot discard the existence of missing targets (\$ 4 ) and, if so, the figures we provide have to be scaled up accordingly. Although it is difficult to tell how much, we will try to make an educated guess. About sixty known XMP galaxies in the SDSS field of view have not been included in our selection (see Fig. 6). They may have been excluded for several reasons because they are too faint, because noise in the spectra artificially enhances [NII] $\lambda 6583$ with respect to $\mathrm{H} \alpha$, because [NII] $\lambda 6583$ exceeds the limits of our selection, and possibly others. Since some of them are proper reasons for exclusion, the number of sixty undetected galaxies may be taken as an upper limit for those missing. Consequently, the number densities we estimate may need to be increased, but only up to a factor of three. 

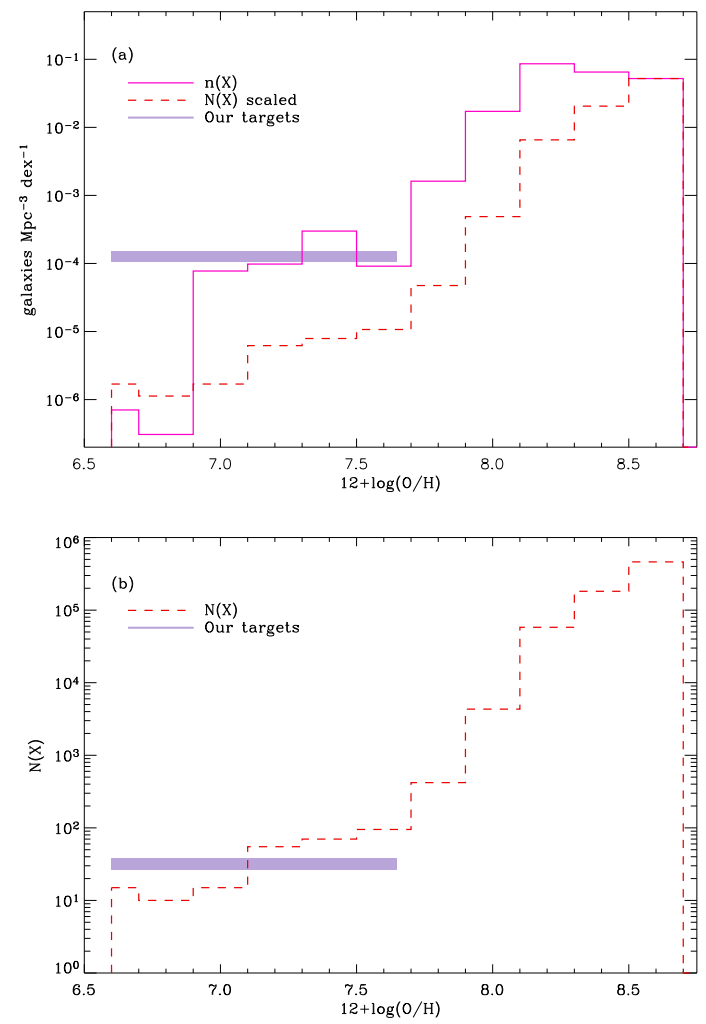

Fig. 8.- (a) Volume number density of galaxies with a given metallicity as inferred from the SDSS/DR7 galaxies with emission lines $(n[X]$, the solid line). The bar represents the average value inferred from the 32 XMP galaxies identified in this study. (b) Histogram of galaxies with a given metallicity as directly observed in SDSS/DR7 (the dashed line). A scaled up version of this histogram is also shown as the dashed line in (a). Note how the already scarce XMP galaxies, represented by the solid line, are hindered even further from observation because they are dwarf, and so, only observable in our neighborhood. 


\section{Conclusions}

We have carried out a systematic search for extremely metal poor (XMP) galaxies among the spectroscopic sample of SDSS/DR7. These objects are rare, and have a clear cosmological interest as unevolved galaxies probably tracing physical conditions in an early phase of the universe (see $\S$ 1). The search is based on the classification of a narrow spectral region around $\mathrm{H} \alpha$, known to be particularly sensitive to the (HII gas) metallicity. Obviously, the almost one million spectra in the database cannot be inspected individually, and we resort to the use of a standard automatic method of classification: k-means. After two nested runs of the procedure, and a subsequent cleaning up for artifacts created by the SDSS pipeline, we end up with 32 targets ( $\S 2$, Table 1). They represent only $0.01 \%$ of the observed galaxies with emission lines. The final metallicity estimate remains pending, however, strong-line empirical calibrations by Pettini \& Pagel (2004) imply their oxygen metallicity to be of the order of one-tenth solar. Obviously, our candidates must be studied in detail through imaging and spectroscopy in following up work.

In order to put the work into context, we carried out a bibliographic search for galaxies with metallicity smaller than one-tenth the solar value (§ 4). We find only 129 (Table 2), and only 21 of them overlap with our sample which, consequently, provides 11 new XMP candidates. The oxygen metallicity of the 21 known targets turns out to be $12+\log (\mathrm{O} / \mathrm{H}) \simeq$ $7.61 \pm 0.19$. These metallicities are based on electron temperatures and, thus, they are more precise than the empirical calibrations we have been employing. Assuming this subset to be representative of the full sample, it confirms the XMP character of our targets.

Our procedure is systematic, therefore, in principle, it should have identified all the spectra in SDSS/DR7 where $\mathrm{H} \alpha \gg[\mathrm{NII}] \lambda 6583$. This assumption, together with the fact that the SDSS spectroscopic sample is limited in apparent magnitude, allows us to estimate the volume number density of XMP galaxies. Using the $V_{\max }$ approximation, we estimate it to be $(1.32 \pm 0.23) \cdot 10^{-4} \mathrm{Mpc}^{-3}$. So far as we are aware of, it provides the first estimate of this number density. The XMP galaxies represent $0.1 \%$ of the galaxies in the local volume, or $\sim 0.2 \%$ considering only emission line galaxies.

We analyze some of the physical properties of the candidates in $\S$ 3. All but four of our XMP candidates turn out to be blue compact dwarfs (BCDs). Note that this association is by no means trivial. We have selected our sample according to the shape of their spectra in an narrow spectral window around $\mathrm{H} \alpha$, and this narrow bit of spectrum turns out to determine many global properties of the galaxy such as color, compactness, and star formation rate. We ignore what causes the association between XMPs and BCDs. The fact that most metal poor galaxies tend to be BCDs is known. Kunth \& Östlin (2000) point it out, but warn against a trivial interpretation since it may reflect an observational bias that makes it easier 
to detect high surface brightness objects such as BCDs. Actually, there are XMP galaxies whose surface brightness does not suffice to call them BCDs (see $\S$ 4). However, the fact that our XMP candidates are also BCDs is revealing. The surface brightness where SDSS starts having problems of completeness is about $\mu_{g}>23.5$ (Blanton et al. 2005), i.e., one magnitude fainter than the faintest XMP candidate. Consequently, if we do not find low surface brightness galaxies with $[\mathrm{NII}] \lambda 6583 \ll \mathrm{H} \alpha$ is because they do not exist in SDSS/DR7.

Among the 32 XMP candidates, 24 of them have either cometary shape or are formed by chains of knots. Papaderos et al. (2008) already noticed the trend for XMP BCDs to reveal a cometary morphology due to the presence of intense star formation at one edge that gradually decreases. This shape is not unique to XMP galaxies, but it comprises only $10 \%$ of all BCDs (Loose \& Thuan 1985), whereas it is dominant when they are metal poor (Papaderos et al. 2008). The origin of the XMP shapes is also unclear. There are arguments for gravitational triggering due to mergers with low-mass stellar or gaseous companions, or for the self propagation of star formation activity within a pre-existing gas rich galaxy (Papaderos et al. 2008). Recently Ekta \& Chengalur (2010a) and Ekta et al. (2008) have found that all XMP galaxies have distorted HI morphologies, which may indicate the infall of external unenriched gas feeding the starburst and dropping the metallicity (e.g., Kewley et al. 2006). It may also be the signature of gas stripping forced by the interaction with an external medium (e.g., Gavazzi et al. 2001; Elmegreen \& Elmegreen 2010).

A concluding remark is in order. The methods for mining massive astronomical databases are still under development. The bases are simply too large for the traditional techniques to be efficient. In this sense, the current paper presents a new approach that may be of interest beyond our particular application. The standard method to find XMP galaxies (or galaxies with any other property) would have been to set, beforehand, the observational criteria the targets should fulfill. Then those targets complying with the criteria would have been selected. Unfortunately, the criteria are often crude and have large uncertainties, which propagate into the selection as false objects sneaking in or true objects leaking out. Here the criteria have not been stipulated in advance. We classify the whole database, and only afterward we select those galaxies belonging the classes that have the intended property. The search is comprehensive, and the results are robust against uncertainties in the selection criteria.

Thanks are due to B. Elmegreen for fruitful discussions on the origin of the cometary shapes in XMP galaxies. Thanks are also due to an anonymous referee for helping us completing the bibliographic search, as well as for insightful comments that prompted an improvement of both the argumentation and the presentation. This work has been partly funded by the Spanish MICINN, projects AYA 2007-67965-03-01, AYA 2007-67752-C03-01, 
and AYA 2010-21887-C04-04. JSA, ALA and CMT are members of the Consolider-Ingenio 2010 Program, grant MICINN CSD2006-00070: First Science with GTC. Funding for the SDSS and SDSS-II has been provided by the Alfred P. Sloan Foundation, the Participating Institutions, the National Science Foundation, the U.S. Department of Energy, the National Aeronautics and Space Administration, the Japanese Monbukagakusho, the Max Planck Society, and the Higher Education Funding Council for England. The SDSS is managed by the Astrophysical Research Consortium for the Participating Institutions (for details, see the SDSS web site at http://www.sdss.org/).

Facilities: Sloan (DR7, spectra)

\section{REFERENCES}

Abazajian, K. N., Adelman-McCarthy, J. K., Agüeros, M. A., et al. 2009, ApJS, 182, 543

Amorín, R., Aguerri, J. A. L., Muñoz-Tuñón, C., \& Cairós, L. M. 2009, A\&A, 501, 75

Amorín, R. O., Muñoz-Tuñón, C., Aguerri, J. A. L., Cairós, L. M., \& Caon, N. 2007, A\&A, 467,541

Asplund, M. 2005, ARA\&A, 43, 481

Baldwin, J. A., Phillips, M. M., \& Terlevich, R. 1981, PASP, 93, 5

Balogh, M. L., Pearce, F. R., Bower, R. G., \& Kay, S. T. 2001, MNRAS, 326, 1228

Bishop, C. M. 2006, Pattern Recognition and Machine Learning (NY: Springer)

Blanton, M. R., Hogg, D. W., Bahcall, N. A., et al. 2003, ApJ, 592, 819

Blanton, M. R., Lupton, R. H., Schlegel, D. J., et al. 2005, ApJ, 631, 208

Blanton, M. R. \& Moustakas, J. 2009, ARA\&A, 47, 159

Bromm, V. \& Larson, R. B. 2004, ARA\&A, 42, 79

Brown, W. R., Kewley, L. J., \& Geller, M. J. 2008, AJ, 135, 92

Cairós, L. M., Caon, N., Vílchez, J. M., González-Pérez, J. N., \& Muñoz-Tuñón, C. 2001a, ApJS, 136, 393

Cairós, L. M., Caon, N., Zurita, C., et al. 2010, A\&A, 520, A90+ 
Cairós, L. M., Vílchez, J. M., González Pérez, J. N., Iglesias-Páramo, J., \& Caon, N. 2001b, ApJS, 133, 321

Denicoló, G., Terlevich, R., \& Terlevich, E. 2002, MNRAS, 330, 69

Diemand, J., Kuhlen, M., \& Madau, P. 2007, ApJ, 657, 262

Ekta, Chengalur, J. N., \& Pustilnik, S. A. 2008, MNRAS, 391, 881

Ekta, B. \& Chengalur, J. N. 2010a, MNRAS, 403, 295

Ekta, B. \& Chengalur, J. N. 2010b, MNRAS, 406, 1238

Ellison, S. L., Songaila, A., Schaye, J., \& Pettini, M. 2000, AJ, 120, 1175

Elmegreen, B. G. \& Elmegreen, D. M. 2010, ApJ, 722, 1895

Everitt, B. S. 1995, Cluster Analysis (London: Arnold)

Gavazzi, G., Boselli, A., Mayer, L., et al. 2001, ApJ, 563, L23

Gil de Paz, A., Madore, B. F., \& Pevunova, O. 2003, ApJS, 147, 29

Griffith, R. L., Tsai, C.-W., Stern, D., et al. 2011, ArXiv e-prints

Guseva, N. G., Izotov, Y. I., Papaderos, P., et al. 2001, A\&A, 378, 756

Guseva, N. G., Izotov, Y. I., Papaderos, P., \& Fricke, K. J. 2007, A\&A, 464, 885

Guseva, N. G., Papaderos, P., Izotov, Y. I., et al. 2003a, A\&A, 407, 75

Guseva, N. G., Papaderos, P., Izotov, Y. I., et al. 2003b, A\&A, 407, 91

Guseva, N. G., Papaderos, P., Izotov, Y. I., et al. 2003c, A\&A, 407, 105

Guseva, N. G., Papaderos, P., Meyer, H. T., Izotov, Y. I., \& Fricke, K. J. 2009, A\&A, 505, 63

Hidalgo-Gámez, A. M. \& Olofsson, K. 2002, A\&A, 389, 836

Izotov, Y. I., Guseva, N. G., Fricke, K. J., \& Papaderos, P. 2009, A\&A, 503, 61

Izotov, Y. I., Guseva, N. G., \& Thuan, T. X. 2011, ApJ, 728, 161

Izotov, Y. I., Papaderos, P., Guseva, N. G., Fricke, K. J., \& Thuan, T. X. 2004, A\&A, 421, 539 
Izotov, Y. I., Papaderos, P., Guseva, N. G., Fricke, K. J., \& Thuan, T. X. 2006a, A\&A, 454, 137

Izotov, Y. I., Stasińska, G., Meynet, G., Guseva, N. G., \& Thuan, T. X. 2006b, A\&A, 448, 955

Izotov, Y. I. \& Thuan, T. X. 2002, ApJ, 567, 875

Izotov, Y. I. \& Thuan, T. X. 2004a, ApJ, 616, 768

Izotov, Y. I. \& Thuan, T. X. 2004b, ApJ, 602, 200

Izotov, Y. I. \& Thuan, T. X. 2007, ApJ, 665, 1115

Izotov, Y. I., Thuan, T. X., \& Guseva, N. G. 2005, ApJ, 632, 210

Izotov, Y. I., Thuan, T. X., \& Guseva, N. G. 2007a, ApJ, 671, 1297

Izotov, Y. I., Thuan, T. X., \& Stasińska, G. 2007b, ApJ, 662, 15

Jones, D. H., Saunders, W., Colless, M., et al. 2004, MNRAS, 355, 747

Kauffmann, G., Heckman, T. M., Tremonti, C., et al. 2003, MNRAS, 346, 1055

Kewley, L. J., Geller, M. J., \& Barton, E. J. 2006, AJ, 131, 2004

Klypin, A., Kravtsov, A. V., Valenzuela, O., \& Prada, F. 1999, ApJ, 522, 82

Kniazev, A. Y., Grebel, E. K., Hao, L., et al. 2003, ApJ, 593, L73

Kniazev, A. Y., Grebel, E. K., Pustilnik, S. A., Pramskij, A. G., \& Zucker, D. B. 2005, AJ, 130,1558

Kniazev, A. Y. \& Pustil'Nik, S. A. 1998, Bull. Special Astrophys. Obs., 46, 23

Kniazev, A. Y., Pustilnik, S. A., Masegosa, J., et al. 2000, A\&A, 357, 101

Kniazev, A. Y., Pustilnik, S. A., Ugryumov, A. V., \& Pramsky, A. G. 2001, A\&A, 371, 404

Kunth, D. \& Östlin, G. 2000, A\&A Rev., 10, 1

Kunth, D. \& Sargent, W. L. W. 1986, ApJ, 300, 496

Lee, H., Grebel, E. K., \& Hodge, P. W. 2003, A\&A, 401, 141

Lee, H., Skillman, E. D., Cannon, J. M., et al. 2006, ApJ, 647, 970 
Lee, J. C., Salzer, J. J., \& Melbourne, J. 2004, ApJ, 616, 752

Lequeux, J., Peimbert, M., Rayo, J. F., Serrano, A., \& Torres-Peimbert, S. 1979, A\&A, 80, 155

Lilly, S. J., Carollo, C. M., \& Stockton, A. N. 2003, ApJ, 597, 730

Loose, H. \& Thuan, T. X. 1985, in Star-forming Dwarf Galaxies and Related Objects, ed. D. Kunth, T. X. Thuan, \& J. Tran Thanh Van (Gif sur Yvette: Editions Frontières), 73

Malmberg, D. 2005, Master's thesis, Uppsala University, Uppsala

Masegosa, J., Moles, M., \& Campos-Aguilar, A. 1994, ApJ, 420, 576

Nagao, T., Maiolino, R., \& Marconi, A. 2006, A\&A, 459, 85

Nava, A., Casebeer, D., Henry, R. B. C., \& Jevremovic, D. 2006, ApJ, 645, 1076

Pagel, B. E. J., Simonson, E. A., Terlevich, R. J., \& Edmunds, M. G. 1992, MNRAS, 255, 325

Papaderos, P., Guseva, N. G., Izotov, Y. I., \& Fricke, K. J. 2008, A\&A, 491, 113

Papaderos, P., Guseva, N. G., Izotov, Y. I., et al. 2006, A\&A, 457, 45

Peimbert, M. \& Torres-Peimbert, S. 1974, ApJ, 193, 327

Pérez-Montero, E. \& Contini, T. 2009, MNRAS, 398, 949

Pettini, M. \& Pagel, B. E. J. 2004, MNRAS, 348, L59

Pilyugin, L. S. 2001, A\&A, 374, 412

Popescu, C. C. \& Hopp, U. 2000, A\&AS, 142, 247

Pustilnik, S., Kniazev, A., Pramskij, A., et al. 2004, A\&A, 419, 469

Pustilnik, S. A., Engels, D., Kniazev, A. Y., et al. 2006, Astronomy Letters, 32, 228

Pustilnik, S. A., Engels, D., Lipovetsky, V. A., et al. 2005, A\&A, 442, 109

Pustilnik, S. A. \& Martin, J.-M. 2007, A\&A, 464, 859

Pustilnik, S. A., Martin, J.-M., Huchtmeier, W. K., et al. 2002, A\&A, 389, 405 
Pustilnik, S. A., Tepliakova, A. L., Kniazev, A. Y., \& Burenkov, A. N. 2008, Astrophysical Bulletin, 63, 102

Pustilnik, S. A., Tepliakova, A. L., Kniazev, A. Y., Martin, J.-M., \& Burenkov, A. N. 2010, MNRAS, 401, 333

Roennback, J. \& Bergvall, N. 1995, A\&A, 302, 353

Sánchez Almeida, J., Aguerri, J. A., Muñoz-Tuñón, C., \& de Vicente, A. 2010, ApJ, 714, 487

Sánchez Almeida, J., Aguerri, J. A., Muñoz-Tuñón, C., \& Vazdekis, A. 2009, ApJ, 698, 1497

Sánchez Almeida, J. \& Lites, B. W. 2000, ApJ, 532, 1215

Sánchez Almeida, J., Muñoz-Tuñón, C., Amorín, R., et al. 2008, ApJ, 685, 194

Sargent, W. L. W. \& Searle, L. 1970, ApJ, 162, L155

Schmidt, M. 1968, ApJ, 151, 393

Shi, F., Kong, X., Li, C., \& Cheng, F. Z. 2005, A\&A, 437, 849

Skillman, E. D., Côté, S., \& Miller, B. W. 2003, AJ, 125, 610

Skillman, E. D., Kennicutt, R. C., \& Hodge, P. W. 1989, ApJ, 347, 875

Spite, M. \& Spite, F. 1982, Nature, 297, 483

Stasińska, G. 2004, in Cosmochemistry. The melting pot of the elements, ed. C. Esteban, R. García López, A. Herrero, \& F. Sánchez (Cambridge: CUP), 115

Stoughton, C., Lupton, R. H., Bernardi, M., et al. 2002a, AJ, 123, 485

Stoughton, C., Lupton, R. H., Bernardi, M., et al. 2002b, AJ, 123, 485

Takeuchi, T. T., Yoshikawa, K., \& Ishii, T. T. 2000, ApJS, 129, 1

Thuan, T. X. \& Izotov, Y. I. 2005, ApJS, 161, 240

Tremonti, C. A., Heckman, T. M., Kauffmann, G., et al. 2004, ApJ, 613, 898

van Zee, L. \& Haynes, M. P. 2006, ApJ, 636, 214

van Zee, L., Skillman, E. D., \& Haynes, M. P. 2006, ApJ, 637, 269 
Vílchez, J. M. \& Iglesias-Páramo, J. 2003, ApJS, 145, 225

Viticchié, B. \& Sánchez Almeida, J. 2011, A\&A, 530, A14+

Yin, S. Y., Liang, Y. C., Hammer, F., et al. 2007, A\&A, 462, 535 